\title{
Implicit-explicit (IMEX) Runge-Kutta methods for non-hydrostatic atmospheric models
}

\author{
David J. Gardner ${ }^{1}$, Jorge E. Guerra ${ }^{2}$, François P. Hamon ${ }^{3}$, Daniel R. Reynolds ${ }^{4}$, Paul A. Ullrich ${ }^{2}$, and \\ Carol S. Woodward ${ }^{1}$ \\ ${ }^{1}$ Center for Applied Scientific Computing, Lawrence Livermore National Laboratory, 7000 East Ave., \\ Livermore, CA 94550, USA \\ ${ }^{2}$ Department of Land, Air and Water Resources, University of California, Davis, One Shields Ave., Davis, CA 95616, USA \\ ${ }^{3}$ Center for Computational Sciences and Engineering, Lawrence Berkeley National Laboratory, \\ 1 Cyclotron Rd., Berkeley, CA 94720 \\ ${ }^{4}$ Department of Mathematics, Southern Methodist University, P.O. Box 750156, Dallas, TX 75257, USA
}

Correspondence: David J. Gardner (gardner48@1lnl.gov)

Received: 8 November 2017 - Discussion started: 9 November 2017

Revised: 1 February 2018 - Accepted: 2 February 2018 - Published: 17 April 2018

\begin{abstract}
The efficient simulation of non-hydrostatic atmospheric dynamics requires time integration methods capable of overcoming the explicit stability constraints on time step size arising from acoustic waves. In this work, we investigate various implicit-explicit (IMEX) additive Runge-Kutta (ARK) methods for evolving acoustic waves implicitly to enable larger time step sizes in a global non-hydrostatic atmospheric model. The IMEX formulations considered include horizontally explicit - vertically implicit (HEVI) approaches as well as splittings that treat some horizontal dynamics implicitly. In each case, the impact of solving nonlinear systems in each implicit ARK stage in a linearly implicit fashion is also explored.

The accuracy and efficiency of the IMEX splittings, ARK methods, and solver options are evaluated on a gravity wave and baroclinic wave test case. HEVI splittings that treat some vertical dynamics explicitly do not show a benefit in solution quality or run time over the most implicit HEVI formulation. While splittings that implicitly evolve some horizontal dynamics increase the maximum stable step size of a method, the gains are insufficient to overcome the additional cost of solving a globally coupled system. Solving implicit stage systems in a linearly implicit manner limits the solver cost but this is offset by a reduction in step size to achieve the desired accuracy for some methods. Overall, the third-order ARS343 and ARK324 methods performed the best, followed by the second-order ARS232 and ARK232 methods.
\end{abstract}

\section{Introduction}

Present-day global climate simulations typically use an atmospheric model configured with a horizontal resolution greater than $10 \mathrm{~km}$. At these scales, the equations governing atmospheric motion can utilize the hydrostatic approximation, which assumes a balance between the gravitational and vertical pressure gradient forces and neglects terms related to vertical acceleration and transport of vertical momentum. As a consequence of this simplification, vertically propagating sound waves, which are of little significance in climate studies, are eliminated from the model. This practice is advantageous for computational efficiency with fully explicit time stepping methods, as vertical sound waves impose a stricter stability limit on step size than horizontal sound waves due to the high horizontal to vertical aspect ratio of the mesh. With the most significant constraint on step size removed, explicit approaches are an attractive option despite their step size limitations from horizontal sound waves. Explicit approaches are employed because of their ease of implementation, the locality of computations, and minimal parallel communication. However, in the near future, increased computational power will enable global climate simulations at scales beyond the hydrostatic limit where vertical acceleration cannot be ignored. At these high resolutions, new model formulations and numerical methods are needed in order to overcome the computational limitations arising from the fastest waves in the atmosphere. 
Accurately modeling atmospheric phenomena at horizontal resolutions below $10 \mathrm{~km}$ necessitates moving to a nonhydrostatic formulation of the governing equations. The step size constraints from sound waves can be addressed either by removing the fast waves in the model with a soundproof formulation of the equations or using a numerical method that can stably step over the fastest waves. The latter approach includes split-explicit (e.g., Klemp et al., 2007), implicitexplicit (IMEX) (e.g., Ullrich and Jablonowski, 2012), and fully implicit (e.g., Yang and Cai, 2014; Yang et al., 2016) time stepping methods. Fully implicit methods enable time steps sizes dictated by the timescales of the processes of interest rather than the stability of the fastest propagating waves. However, achieving good scalability with these methods can be quite challenging as they require optimized nonlinear solvers and preconditioners to efficiently compute the solution of globally coupled nonlinear systems. As such, split-explicit and IMEX approaches present a potentially simpler alternative as only a subset of the dynamics is treated implicitly. These approaches allow for specialized solvers that can exploit properties of the implicit system at the cost of being stability limited by the fastest waves in the explicit portion of the splitting.

Split-explicit methods typically divide the dynamics into three groups: fast vertical waves that are treated implicitly, fast horizontal waves that are substepped relative to the other dynamics with an explicit method, and slow dynamics that are updated with an explicit method using a long time step (e.g., Klemp et al., 2007). Similarly, IMEX methods partition the dynamics into two parts: non-stiff terms that are explicitly updated and stiff terms that are implicitly solved. In a horizontally explicit - vertically implicit (HEVI) IMEX approach, all horizontal motion is treated explicitly and vertical dynamics are updated implicitly. By solving only the vertical dynamics implicitly, both split-explicit and HEVI methods take advantage of the two-dimensional horizontal domain decomposition of atmospheric models to avoid communication between parallel processes. Since each process owns a subset of the vertical columns in the global domain, no message passing is necessary during the vertically implicit solves. Split-explicit approaches are able to gain some additional efficiency by substepping the fast horizontal terms while step sizes with HEVI methods are limited by the fastest horizontal dynamics. This restriction can be overcome by incorporating some horizontal terms into the implicit partition at the cost of solving a larger, globally coupled system requiring interprocessor communication during the implicit solve. If the increase in stable step size is sufficiently large, these methods may be able to overcome the additional expense from parallel communication with an efficient nonlinear solver.

With the push toward exascale computing, there has been increasing interest in evaluating the potential of IMEX methods for efficiently simulating atmospheric dynamics at high resolution. Ullrich and Jablonowski (2012) presented results using a Runge-Kutta-Rosenbrock and Strang carry- over IMEX approach for integrating a non-hydrostatic model in Cartesian geometry. A new second-order Runge-Kutta IMEX method is presented in Giraldo et al. (2013) and compared with Runge-Kutta and multistep IMEX integration schemes from the literature in the non-hydrostatic unified model of the atmosphere (NUMA). Using NUMA, the accuracy and efficiency of the integration methods were evaluated with a one-dimensional linear HEVI splitting and three-dimensional linear IMEX splitting by simulating a two-dimensional rising thermal bubble and inertia-gravity waves in three-dimensional Cartesian and spherical domains. Weller et al. (2013) examined the stability properties of 12 Runge-Kutta IMEX methods and compared the accuracy of the methods with two HEVI splittings against a semi-implicit approach using the two-dimensional compressible Boussinesq equations. The work of Lock et al. (2014) performs a detailed analysis of the same IMEX methods from Weller et al. (2013) on linear scalar and two-dimensional wave equations.

In this work, we investigate the performance of 21 RungeKutta IMEX methods from the literature, including many of those tested in Ullrich and Jablonowski (2012), Giraldo et al. (2013), Weller et al. (2013), and Lock et al. (2014), on a non-hydrostatic atmospheric dynamical core using different implicit-explicit splittings of the governing equations and approaches for solving the nonlinear systems. Methods tested in Weller et al. (2013) and Lock et al. (2014) are evaluated with a three-dimensional fully compressible set of governing equations that differs from those considered in Giraldo et al. (2013) in terms of formulation, discretization, and approach to implicit-explicit splitting. The Runge-Kutta methods tested in Giraldo et al. (2013) are also included in this study along with additional methods from the literature not considered in the previously cited works. The linearly implicit Runge-Kutta-Rosenbrock approach utilized in Ullrich and Jablonowski (2012) is also compared against a Newton iteration for solving the nonlinear systems that arise in each implicit Runge-Kutta stage.

The choices of IMEX partitioning, integration method, and implicit solver are evaluated in terms of accuracy and efficiency using the Tempest non-hydrostatic dynamical core (Ullrich, 2014) to determine the optimal combination. Tempest is a flexible global modeling framework for evaluating numerical methods for next-generation, high-resolution climate simulations on high-performance computing systems. To ease the exploration of a wide variety of splitting choices and integration schemes, we have interfaced Tempest with the ARKode package of additive Runge-Kutta (ARK) methods (Reynolds et al., 2018) from the SUNDIALS library (Hindmarsh et al., 2005; SUNDIALS, 2017) of algebraic and differential equations solvers. ARKode is an adaptive-step time integration package for solving initial value problems with fully explicit, fully implicit, or IMEX Runge-Kutta methods. The software framework was designed to be easily incorporated into existing applications and allows signifi- 
cant freedom over the choice of methods and implicit solvers. The versions of Tempest with ARKode interfaces used in this work are archived at Gardner et al. (2017).

In the following section, we present the formulation of non-hydrostatic equations implemented in Tempest, followed by a discussion of the spatial and temporal discretizations and splitting approaches in Sect. 3. The nonlinear and linear solver strategies used with the IMEX methods are covered in Sect. 4. Numerical experiments and the corresponding results are given in Sect. 5. A summary of the numerical results, concluding remarks, and directions for future work are given in Sect. 6.

\section{Non-hydrostatic equations}

The non-hydrostatic dry-atmosphere/shallow-atmosphere equations in the Tempest $\theta$ formulation are in terms of covariant horizontal velocities $u_{\alpha}$ and $u_{\beta}$, covariant vertical velocity $u_{\xi}$, potential temperature $\theta$, and density $\rho$ in an arbitrary coordinate system $(\alpha, \beta, \xi)$ :

$$
\begin{aligned}
\frac{\partial u_{\alpha}}{\partial t}= & -\frac{\partial}{\partial \alpha}(K+\Phi)-\theta \frac{\partial \Pi}{\partial \alpha} \\
& +J u^{\beta}\left(f r_{\xi}^{-1}+\zeta^{\xi}\right)-J u^{\xi} \zeta^{\beta}, \\
\frac{\partial u_{\beta}}{\partial t}= & -\frac{\partial}{\partial \beta}(K+\Phi)-\theta \frac{\partial \Pi}{\partial \beta} \\
& -J u^{\alpha}\left(f r_{\xi}^{-1}+\zeta^{\xi}\right)+J u^{\xi} \zeta^{\alpha}, \\
\frac{\partial u \xi}{\partial t}= & -\frac{\partial}{\partial \xi}(K+\Phi)-\theta \frac{\partial \Pi}{\partial \xi}+J\left(u^{\alpha} \zeta^{\beta}-u^{\beta} \zeta^{\alpha}\right), \\
\frac{\partial \theta}{\partial t}= & -u^{\alpha} \frac{\partial \theta}{\partial \alpha}-u^{\beta} \frac{\partial \theta}{\partial \beta}-u^{\xi} \frac{\partial \theta}{\partial \xi}, \\
\frac{\partial \rho}{\partial t}= & -\frac{1}{J} \frac{\partial}{\partial \alpha}\left(J \rho u^{\alpha}\right)-\frac{1}{J} \frac{\partial}{\partial \beta}\left(J \rho u^{\beta}\right) \\
& -\frac{1}{J} \frac{\partial}{\partial \xi}\left(J \rho u^{\xi}\right) .
\end{aligned}
$$

We refer to the system defined by Eqs. (1)-(5) as the $\theta$ formulation of the non-hydrostatic equations since the thermodynamic equation (Eq. 4) is expressed in terms of the potential temperature. The conversion between contravariant and covariant velocity components is given as

$u_{i}=g_{i \alpha} u^{\alpha}+g_{i \beta} u^{\beta}+g_{i \xi} u^{\xi}$

$u^{i}=g^{i \alpha} u_{\alpha}+g^{i \beta} u_{\beta}+g^{i \xi} u_{\xi}$,

where $g_{i j}$ and $g^{i j}$ are the covariant and contravariant metric tensors specified in terrain-following Cartesian or spherical coordinates and $J$ is the metric Jacobian defined as

$J=\sqrt{\operatorname{det} g_{i j}}$.

$\Phi$ is the product of the gravity constant and elevation $r$, $r_{\xi}=(\partial r / \partial \xi)$ is the vertical coordinate transform, and $f=$
$2 \Omega \sin \varphi$ is the Coriolis parameter. $K$ is the specific kinetic energy, defined as

$K=\frac{1}{2}\left(u_{\alpha} u^{\alpha}+u_{\beta} u^{\beta}+u_{\xi} u^{\xi}\right)$.

and $\Pi$ is the Exner pressure function defined as

$\Pi=c_{\mathrm{p}}\left(\frac{p_{0}}{p}\right)^{R_{\mathrm{d}} / c_{\mathrm{p}}}=c_{\mathrm{p}}\left(\frac{R_{\mathrm{d}} \rho \theta}{p_{0}}\right)^{R_{\mathrm{d}} / c_{v}}$,

where $R_{\mathrm{d}}, c_{\mathrm{p}}$, and $p_{0}$ are the gas constant for dry air, specific heat at constant pressure, and the reference pressure (here chosen to be $10^{5} \mathrm{~Pa}$ ), respectively. The relative vorticity vector is

$$
\begin{aligned}
\zeta= & \frac{1}{J}\left[\left(\frac{\partial u_{\xi}}{\partial \beta}-\frac{\partial u_{\beta}}{\partial \xi}\right) \boldsymbol{g}_{\alpha}+\left(\frac{\partial u_{\alpha}}{\partial \xi}-\frac{\partial u_{\xi}}{\partial \alpha}\right) \boldsymbol{g}_{\beta}\right. \\
& \left.+\left(\frac{\partial u_{\beta}}{\partial \alpha}-\frac{\partial u_{\alpha}}{\partial \beta}\right) \boldsymbol{g}_{\xi}\right] .
\end{aligned}
$$

\section{Discretization}

The non-hydrostatic equations are discretized using a method of lines approach. First, the terms on the right-hand sides of Eqs. (1)-(5) are discretized in space, and then the resulting system of coupled ordinary differential equations is advanced in time with a numerical integration scheme. This two-step process is detailed in the following sections.

\subsection{Spatial discretization}

The spatial discretization of Eqs. (1)-(5) follows Guerra and Ullrich (2016), where a fourth-order spectral element method is used for horizontal derivatives in $\alpha$ and $\beta$, and the staggered finite element method is used in $\xi$. Unless otherwise stated, test cases in this work will be configured with Lorenz vertical staggering (vertical velocity computed and stored at interfaces including model boundaries) and regular grid distribution in each column.

In Tempest, hyperviscosity is employed in the horizontal directions by default. The operators are fourth-order derivatives with nominal coefficients of $1.0 \times 10^{15} \mathrm{~m}^{2} \mathrm{~s}^{-1}$ following Guerra and Ullrich (2016) and Ullrich (2014). In particular, the use of hyperviscosity corrects dispersive errors and ringing associated with computational modes due to accumulation of energy near the grid truncation scale. The use of hyperviscosity is necessary since the spectral element discretization implicitly conserves energy (Taylor and Fournier, 2010) and hence provides no mechanism for implicit diffusion of energy at short wavelengths. It is important to note that hyperviscosity is applied at the end of each time step as a separate forward Euler update. As such, it is not part of the additive Runge-Kutta method used to integrate the equations. Additionally, vertical upwinding is applied to the horizontal velocities, potential temperature, and density. 


\subsection{Temporal discretization}

There are numerous approaches for integrating the system of ordinary differentia equations (ODEs) resulting from the spatial discretization of Eqs. (1)-(5), including multistep or multistage methods that treat the system in a fully explicit, fully implicit, or split implicit-explicit manner. This work focuses on the application of multistage IMEX integrators defined by ARK methods that split the right-hand side into two parts: an explicit (non-stiff) and an implicit (stiff) part. Section 3.2.1 presents the general formulation of ARK methods. The various options explored for partitioning terms in the non-hydrostatic equations into implicit and explicit parts are presented in Sect. 3.2.2 and 3.2.3.

\subsubsection{Additive Runge-Kutta methods}

The spatially discretized non-hydrostatic equations can be written as a general initial value problem with the right-hand side additively split into two parts:

$\frac{\mathrm{d} \boldsymbol{y}}{\mathrm{d} t}=\boldsymbol{f}^{\mathrm{E}}(t, \boldsymbol{y})+\boldsymbol{f}^{\mathrm{I}}(t, \boldsymbol{y}), \quad \boldsymbol{y}\left(t_{0}\right)=\boldsymbol{y}_{0}$.

The model state vector is $\boldsymbol{y}=\left(u_{\alpha}, u_{\beta}, w, \theta, \rho\right)^{\mathrm{T}}$ in the $\theta$ formulation. Under this notation, $f^{\mathrm{E}}$ and $\boldsymbol{f}^{\mathrm{I}}$ correspond to the spatial terms that will be integrated explicitly and implicitly, respectively, and $\boldsymbol{y}_{0}$ is the initial state at time $t_{0}$. The system (Eq. 10) is evolved from time $t_{n-1}$ to time $t_{n}$ using ARK methods of the form

$$
\begin{aligned}
z_{i}= & \boldsymbol{y}_{n-1}+h_{n} \sum_{j=1}^{i-1} a_{i, j}^{\mathrm{E}} \boldsymbol{f}^{\mathrm{E}}\left(t_{n, j}^{\mathrm{E}}, z_{j}\right) \\
& +h_{n} \sum_{j=1}^{i} a_{i, j}^{\mathrm{I}} f^{\mathrm{I}}\left(t_{n, j}^{\mathrm{I}}, z_{j}\right), \quad i=1, \ldots, s \\
\boldsymbol{y}_{n}= & \boldsymbol{y}_{n-1}+h_{n} \sum_{i=1}^{s}\left(b_{i}^{\mathrm{E}} \boldsymbol{f}^{\mathrm{E}}\left(t_{n, i}^{\mathrm{E}}, z_{i}\right)+b_{i}^{\mathrm{I}} \boldsymbol{f}^{\mathrm{I}}\left(t_{n, i}^{\mathrm{I}}, z_{i}\right)\right),
\end{aligned}
$$

where $\boldsymbol{y}_{n}$ is an approximation of $\boldsymbol{y}\left(t_{n}\right), \boldsymbol{z}_{i}$ is an intermediate stage solution in an ARK method with $s$ stages, $h_{n}=$ $t_{n}-t_{n-1}$ is the time step size, and $t_{n, i}^{\mathrm{E}}=t_{n-1}+c_{i}^{\mathrm{E}} h_{n}$ and $t_{n, i}^{\mathrm{I}}=t_{n-1}+c_{i}^{\mathrm{I}} h_{n}$ are intermediate stage times. Several of the methods we examine include an embedded solution,

$\widetilde{\boldsymbol{y}}_{n}=\boldsymbol{y}_{n-1}+h_{n} \sum_{i=1}^{s}\left(\widetilde{b}_{i}^{\mathrm{E}} \boldsymbol{f}^{\mathrm{E}}\left(t_{n, i}^{\mathrm{E}}, z_{i}\right)+\widetilde{b}_{i}^{\mathrm{I}} \boldsymbol{f}^{\mathrm{I}}\left(t_{n, i}^{\mathrm{I}}, z_{i}\right)\right)$,

for estimating the local truncation error to adapt the time step size. The numerical studies that follow use a fixed time step size and thus do not utilize a local error estimate. However, methods with embeddings are of particular interest, as we will explore leveraging variable time step sizes in subsequent work.
A particular ARK method is defined by a combination of an explicit and a diagonally implicit pair of Butcher tableaux:

$$
\begin{aligned}
& \begin{array}{c|cc|ccccc} 
& & 0 & 0 & 0 & 0 & \cdots & 0 \\
\boldsymbol{c}^{\mathrm{E}} & \mathbf{A}^{\mathrm{E}} & c_{2}^{\mathrm{E}} & a_{2,1}^{\mathrm{E}} & 0 & 0 & \cdots & 0 \\
& c_{3}^{\mathrm{E}} & a_{3,1}^{\mathrm{E}} & a_{3,2}^{\mathrm{E}} & 0 & \cdots & 0 \\
& \boldsymbol{b}^{\mathrm{E}}= & \vdots & & & \ddots & 0 \\
& \widetilde{\boldsymbol{b}}^{\mathrm{E}} & c_{s}^{\mathrm{E}} & a_{s, 1}^{\mathrm{E}} & a_{s, 2}^{\mathrm{E}} & \cdots & a_{s, s-1}^{\mathrm{E}} & 0 \\
\hline & & b_{1}^{\mathrm{E}} & b_{2}^{\mathrm{E}} & \cdots & b_{s-1}^{\mathrm{E}} & b_{s}^{\mathrm{E}} \\
& & \widetilde{b}_{1}^{\mathrm{E}} & \widetilde{b}_{2}^{\mathrm{E}} & \cdots & \widetilde{b}_{s-1}^{\mathrm{E}} & \widetilde{b}_{s}^{\mathrm{E}}
\end{array}
\end{aligned}
$$

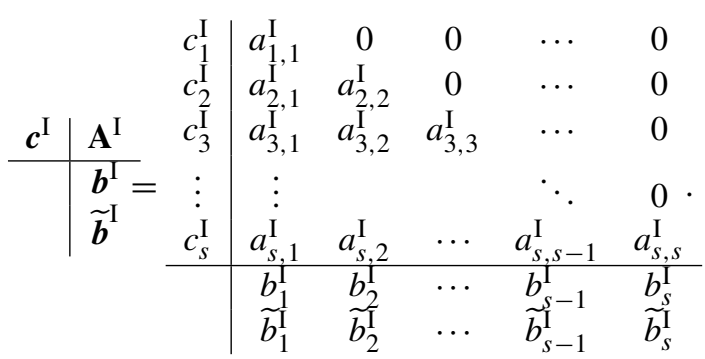

When $a_{i, i}^{\mathrm{I}} \neq 0$, computing the stage value $z_{i}$ requires solving a (non)linear system of equations. This system and approaches for computing the stage solutions are discussed in Sect. 4.

While Eqs. (11)-(13) define an ARK method in terms of a linear combination of right-hand side evaluations at internal stage values, we note that it is customary in the climate modeling community to cast ARK schemes as linear combinations of states produced from explicit and implicit $\mathrm{Eu}-$ ler steps. There, the objective is to store state vectors only and make single explicit/implicit function evaluations at a given stage. Since the stage coefficient matrices in Eq. (14) are lower triangular (strictly so for the explicit coefficients), then any preceding function evaluations may be written in terms of preceding state vectors and substituted into a current stage. While the two approaches are entirely equivalent, we present all ARK methods and their corresponding tables in standard form in Eqs. (11)-(14), both to connect with the literature defining each method, and since we use this form in computing our results.

We investigate a number of ARK methods from the literature with a variety of numerical properties:

- classical second- (ARS232, ARS222, and ARS233) and third-order (ARS343 and ARS443) methods from Ascher et al. (1997);

- the third- (ARK324), fourth- (ARK436), and fifthorder (ARK548) methods from Kennedy and Carpenter (2003);

- the second-order ARK232 method derived for the NUMA model and presented in Giraldo et al. (2013);

- second- (SSP2(222), SSP2(332)a, and SSP3(332)) and third-order (SSP3(433)) strong stability preserving methods from Pareschi and Russo (2005); 
- second-order strong stability preserving method SSP2(332)b from Higueras (2006); the optimized second-order methods SSP2(332)lpm1, SSP2(332)lpm2, SSP2(332)lpum, and SSP2(332)lspum from Higueras et al. (2014); and the third-order method SSP3(333)a from Higueras (2009); and

- third-order strong stability preserving methods SSP3(333)b and SSP3(333)c from Conde et al. (2017).

The ARK232, ARS232, ARS233, ARS443, SSP2(222), SSP2(332)a, SSP3(332), and SSP3(433) methods were previously examined by Weller et al. (2013) with different splittings on two vertical slice cases of the compressible Boussinesq equations. In these tests, the ARK232 method presented in Giraldo et al. (2013) had the best overall performance. Giraldo et al. (2013) also compared ARK232 with ARK324 and ARK436 with different splittings on a 2-D rising thermal bubble test in Cartesian coordinates and 3-D inertia-gravity wave tests in Cartesian coordinates and on the sphere. The ARK324 and ARK436 methods were most efficient when high accuracy was required and ARK232 had greater efficiency when less accuracy was required. In addition to the methods tested in Weller et al. (2013) and Giraldo et al. (2013), we include the ARS222, ARS343, ARK548, and strong stability preserving (SSP) methods from Higueras (2006), Higueras (2009), Higueras et al. (2014), and Conde et al. (2017).

With the exception of ARS233 and SSP3(333)a, b, and c, all of the above methods are constructed with an L stable implicit method. Thus, the implicit portion of the method is accurate in the limit of the stiff term becoming infinitely fast, meaning that slow dynamics are resolved while fast modes, e.g., acoustic waves, are damped. Two methods, ARS233 and SSP2(222), are B stable which is a nonlinear stability indicating that the difference between two numerical solutions does not increase with time. Several methods are SSP and are designed to maintain the total variation diminishing (TVD) property of a spatial discretization. The optimized SSP methods from Higueras et al. (2014) consider additional properties beyond optimizing the region of absolute monotonicity in SSP schemes. A total of 10 of the methods considered are stiffly accurate; that is, $a_{s, i}^{\mathrm{I}}=b_{i}^{\mathrm{I}}$, and two of those methods, ARS222 and ARS443, also have $a_{s, i}^{\mathrm{E}}=b_{i}^{\mathrm{E}}$. Ascher et al. (1997) notes that having the both $a_{s, i}^{\mathrm{I}}=b_{i}^{\mathrm{I}}$ and $a_{s, i}^{\mathrm{E}}=b_{i}^{\mathrm{E}}$ so that $\boldsymbol{z}_{s}=\boldsymbol{y}_{n}$ is useful for very stiff problems. However, it is unclear why this property is beneficial and the two methods with this property do not outperform other methods in the two test cases considered. All methods, except ARS222 and ARS443, have the same $b_{i}$ values for the explicit and implicit methods so that the $f^{\mathrm{E}}$ and $\boldsymbol{f}^{\mathrm{I}}$ functions are weighted equally at the same stage solution. As noted in Kennedy and Carpenter (2003) and Giraldo et al. (2013), ARK methods with $\boldsymbol{b}^{\mathrm{E}}=\boldsymbol{b}^{\mathrm{I}}$ have the desirable property of preserving linear invariants of the problem to machine precision. Many of the methods considered also have the same explicit and implicit stage times. All of the non-SSP methods and the SSP methods of Conde et al. (2017) have $\boldsymbol{c}^{\mathrm{E}}=\boldsymbol{c}^{\mathrm{I}}$, but the other SSP methods tested have different explicit and implicit stage times. Finally, the methods of Kennedy and Carpenter (2003) and Giraldo et al. (2013) have implicit methods with secondorder stage accuracy to limit order reduction when applied to stiff systems. Appendix A contains a summary of different properties of the ARK methods considered in this work.

\subsubsection{Horizontally explicit - vertically implicit splittings}

In this section, we present four HEVI formulations of the non-hydrostatic equations (Eqs. 1-5) in which the horizontal terms are evaluated explicitly and some of the vertical terms are solved implicitly. The partitioning of terms into the explicit or implicit right-hand sides is given by

$$
\begin{aligned}
& \frac{\partial u_{\alpha}}{\partial t}=-\frac{\partial}{\partial \alpha}(K+\Phi)-\theta \frac{\partial \Pi}{\partial \alpha} \\
& +J u^{\beta}\left(f r_{\xi}^{-1}+\zeta^{\xi}\right)-J u^{\xi} \zeta^{\beta}, \\
& \frac{\partial u_{\beta}}{\partial t}=-\frac{\partial}{\partial \beta}(K+\Phi)-\theta \frac{\partial \Pi}{\partial \beta} \\
& -J u^{\alpha}\left(f r_{\xi}^{-1}+\zeta^{\xi}\right)+J u^{\xi} \zeta^{\alpha}, \\
& \frac{\partial u_{\xi}}{\partial t}=\underbrace{-\frac{\partial K}{\partial \xi}+u^{\alpha} \frac{\partial u_{\alpha}}{\partial \xi}+u^{\beta} \frac{\partial u_{\beta}}{\partial \xi}-\frac{\partial \Phi}{\partial \xi}-\theta \frac{\partial \Pi}{\partial \xi}}_{\operatorname{imp~(A,~C)~/~exp~(B,~D)~}} \\
& -u^{\alpha} \frac{\partial u_{\xi}}{\partial \alpha}-u^{\beta} \frac{\partial u_{\xi}}{\partial \beta}, \\
& \frac{\partial \theta}{\partial t}=-u^{\alpha} \frac{\partial \theta}{\partial \alpha}-u^{\beta} \frac{\partial \theta}{\partial \beta} \underbrace{-u^{\xi} \frac{\partial \theta}{\partial \xi}}_{\text {imp (A, B) } / \exp (\mathrm{C}, \mathrm{D})}, \\
& \frac{\partial \rho}{\partial t}=-\frac{1}{J} \frac{\partial}{\partial \alpha}\left(J \rho u^{\alpha}\right)-\frac{1}{J} \frac{\partial}{\partial \beta}\left(J \rho u^{\beta}\right) \\
& \underbrace{-\frac{1}{J} \frac{\partial}{\partial \xi}\left(J \rho u^{\xi}\right)}_{\operatorname{imp~(A,~B,~C,~D)~}} \text {. }
\end{aligned}
$$

The choice of an explicit or implicit treatment of these terms is guided by two core requirements. First, we require that the terms responsible for vertically propagating sound waves (namely, the buoyancy term, $-\frac{\partial \Phi}{\partial \xi}-\theta \frac{\partial \Pi}{\partial \xi}$, in the vertical velocity equation, Eq. 17, and the vertical flux term, $-\frac{1}{J} \frac{\partial}{\partial \xi}\left(J \rho u^{\xi}\right)$, in the density equation, Eq. 19) be handled implicitly. Treating these terms explicitly would leave us bound to the Courant-Friedrichs-Lewy (CFL) condition for vertically propagating sound waves (around $2 \mathrm{~s}$ for the simulations in this paper) and thus would not lead to a computationally competitive scheme. Second, all terms associated 
with vertical momentum transport (the first three terms in Eq. 17) must be handled together. In practice, these terms cancel each other nearly exactly and so splitting them can dramatically impact model stability. These terms can be handled either implicitly or explicitly, as they are associated with the vertical advective speed, and hence an explicit treatment does not impact model stability. Whereas an explicit treatment of these terms is generally simpler, in combination with the last term in Eq. (19) they are together responsible for vertical kinetic energy transport. Consequently, it could be argued that these terms should be handled using the same discretization as the vertical mass transport term in order to ensure energy conservation by the vertically implicit update. In this study, the impact of an explicit and implicit treatment of the vertical transport of $\theta$ is also explored (the last term in Eq. 18). Although sound waves are not expressed in the $\theta$ field, there is a substantial difference in model stability that emerges from whether this term is treated implicitly or explicitly.

Based on the principles above, terms in Eqs. (15)-(19) without underbraces are always evaluated explicitly and those with underbraces are treated either implicitly or explicitly depending on the particular HEVI formulation. In order from most implicit to least implicit, we consider

1. HEVI-A, where all vertical dynamics except vertical advection of horizontal velocity in Eqs. (15) and (16) are treated implicitly;

2. HEVI-B, where vertical velocity advection in Eq. (17) is treated explicitly;

3. HEVI-C, where thermodynamic advection in Eq. (18) is treated explicitly; and

4. HEVI-D, where vertical velocity advection in Eq. (17) and thermodynamic advection in Eq. (18) are treated explicitly.

\subsubsection{IMEX splittings with horizontally implicit terms}

In addition to the HEVI options, we also consider IMEX splittings that solve various parts of horizontal dynamics implicitly. These formulations contain the same vertically implicit terms as HEVI-A but add some of the horizontal terms into the implicit function:

$$
\begin{aligned}
\frac{\partial u_{\alpha}}{\partial t}= & -\frac{\partial}{\partial \alpha}(K+\Phi) \underbrace{-\theta \frac{\partial \Pi}{\partial \alpha}}_{\mathrm{imp}(\mathrm{B}) / \exp (\mathrm{A})} \\
& +J u^{\beta}\left(f r_{\xi}^{-1}+\zeta^{\xi}\right)-J u^{\xi} \zeta^{\beta}, \\
\frac{\partial u_{\beta}}{\partial t}= & -\frac{\partial}{\partial \beta}(K+\Phi) \underbrace{-\theta \frac{\partial \Pi}{\partial \beta}}_{\mathrm{imp}(\mathrm{B}) / \exp (\mathrm{A})} \\
& -J u^{\alpha}\left(f r_{\xi}^{-1}+\zeta^{\xi}\right)+J u^{\xi} \zeta^{\alpha},
\end{aligned}
$$

$$
\begin{aligned}
& \frac{\partial u \xi}{\partial t}=\underbrace{-\frac{\partial}{\partial \xi}(K+\Phi)-\theta \frac{\partial \Pi}{\partial \xi}+u^{\alpha} \frac{\partial u_{\alpha}}{\partial \xi}+u^{\beta} \frac{\partial u_{\beta}}{\partial \xi}}_{\operatorname{imp}(\mathrm{A}, \mathrm{B})} \\
& -u^{\alpha} \frac{\partial u_{\xi}}{\partial \alpha}-u^{\beta} \frac{\partial u_{\xi}}{\partial \beta}, \\
& \frac{\partial \theta}{\partial t}=\underbrace{-u^{\alpha} \frac{\partial \theta}{\partial \alpha}-u^{\beta} \frac{\partial \theta}{\partial \beta}-u^{\xi} \frac{\partial \theta}{\partial \xi}}_{\text {imp(B)/exp(A) }}, \\
& \frac{\partial \rho}{\partial t}=\underbrace{-\frac{1}{J} \frac{\partial}{\partial \alpha}\left(J \rho u^{\alpha}\right)-\frac{1}{J} \frac{\partial}{\partial \beta}\left(J \rho u^{\beta}\right)-\frac{1}{J} \frac{\partial}{\partial \xi}\left(J \rho u^{\xi}\right)}_{\text {imp (A, B })} .
\end{aligned}
$$

As before, terms without underbraces in Eqs. (20)-(24) are treated explicitly. We examine two configurations with horizontally implicit terms:

1. IMEX-A, where the density equation (Eq. 24) is fully implicit, and

2. IMEX-B, where the density (Eq. 24), thermodynamics (Eq. 23), and Exner pressure in Eqs. (20) and (21) are solved implicitly.

The IMEX-A option treats the density equation with a single consistent scheme, while IMEX-B is motivated by semiimplicit splittings (e.g., Weller et al., 2013) and treats the pressure gradient fully implicitly. By incorporating some of the fast horizontal dynamics into the implicit portion of the splitting, these IMEX formulations may enable larger stable step sizes than are possible with the HEVI options. However, treating horizontal dynamics implicitly also introduces coupling between vertical columns in the implicit solves, and this increased coupling in turn increases the linear solution expense. In the numerical experiments below, we will test if the increased steps sizes are enough to offset the additional solver cost.

\section{Solvers}

An $s$-stage ARK method defined by Eqs. (11)-(13) requires the solution of at most $s$ nonlinear systems of the form

$$
\boldsymbol{G}\left(z_{i}\right) \equiv z_{i}-h_{n} a_{i, i}^{\mathrm{I}} \boldsymbol{f}^{\mathrm{I}}\left(t_{n, i}^{\mathrm{I}}, z_{i}\right)-\boldsymbol{d}_{i}=0, \quad i=1, \ldots, s,
$$

to compute the stage solutions, $z_{i}$, where

$$
\boldsymbol{d}_{i} \equiv \boldsymbol{y}_{n-1}+h_{n} \sum_{j=1}^{i-1}\left[a_{i, j}^{\mathrm{E}} \boldsymbol{f}^{\mathrm{E}}\left(t_{n, j}^{\mathrm{E}}, z_{j}\right)+a_{i, j}^{\mathrm{I}} \boldsymbol{f}^{\mathrm{I}}\left(t_{n, j}^{\mathrm{I}}, z_{j}\right)\right]
$$

are known data from previous stage values. The structure of Eq. (25) is highly dependent on the underlying splitting, 
which determines the size of the system and the spatial coupling between the algebraic equations in this system. Therefore, efficient solution strategies that take full advantage of the structure of the nonlinear system resulting from the splitting are highly desirable. This topic is addressed in the following subsections where we present the solver approaches considered in this work.

\subsection{Nonlinear solvers}

Newton's method finds the solution of Eq. (25) using an iterative approach:

$\boldsymbol{z}_{i}^{(m+1)}=\boldsymbol{z}_{i}^{(m)}+\boldsymbol{\delta}^{(m+1)}$,

where $m \geq 0$ is the iteration index and the update $\boldsymbol{\delta}^{(m+1)}$ is the solution of the linear system

$\mathbf{A}\left(z_{i}^{(m)}\right) \boldsymbol{\delta}^{(m+1)}=-\boldsymbol{G}\left(z_{i}^{(m)}\right)$,

obtained from a linearization of Eq. (25),

$\mathbf{A}\left(z_{i}^{(m)}\right) \equiv \mathbf{I}-h_{n} a_{i, i}^{\mathrm{I}} \mathbf{J}\left(z_{i}^{(m)}\right)$,

in which $\mathbf{J}$ is the Jacobian matrix of $\boldsymbol{f}^{\mathrm{I}}$ evaluated at the current iteration. Following the ODE literature, we consider the iteration converged when

$R_{i}\left\|\boldsymbol{\delta}^{(m+1)}\right\|<\epsilon$,

where $R_{i}$ is an estimate of the linear convergence rate, $\|\cdot\|$ is a weighted root mean square (WRMS) norm, and $\epsilon$ is the nonlinear tolerance (Hindmarsh et al., 2005). The convergence rate estimate $R_{i}$ is initialized to 1 and for $m>0$ is updated as

$R_{i}=\max \left(0.3 R_{i}, \frac{\left\|\boldsymbol{\delta}^{(m+1)}\right\|}{\left\|\boldsymbol{\delta}^{(m)}\right\|}\right)$.

For a vector $\boldsymbol{v}$ with length $N$, the WRMS is norm defined as

$\|\boldsymbol{v}\|=\left(\frac{1}{N} \sum_{i=1}^{N}\left(w_{i} v_{i}\right)^{2}\right)^{1 / 2}$, with $w_{i}=\frac{1}{\epsilon_{\mathrm{r}}\left|v_{i}\right|+\epsilon_{\mathrm{a}}}$,

where $\epsilon_{\mathrm{a}}$ and $\epsilon_{\mathrm{r}}$ are the absolute and relative tolerances for the time-evolved solution, respectively. With this choice of weighting, a WRMS norm of 1 is considered small for any error-like quantities since $1 / w_{i}$ represents a tolerance on the components of the solution vector. To keep error in the nonlinear solve from interfering with the time integration error, we use the ARKode default nonlinear tolerance $\epsilon=10^{-1}$ in Eq. (30).

Newton's method can be quite expensive, especially when many iterations are needed to achieve convergence, since each iteration involves computing or approximating the Jacobian matrix and performing a linear solve. As an alternative, we also consider treating Eq. (25) as a linearly implicit system. This Rosenbrock-like approach, used in Ullrich and Jablonowski (2012) and Guerra and Ullrich (2016), consists of performing a single iteration of Newton's method, thus limiting the cost of the nonlinear solver. However, this approach may produce a lower quality solution when one Newton iteration does not sufficiently solve the original nonlinear problem.

In both solver approaches, the solution value $\boldsymbol{y}_{n-1}$ is utilized as the initial iterate, $z_{i}^{0}$ (i.e., the trivial predictor) in the nonlinear solves. While alternative predictor methods are not explored in this work, their impact on the speed and robustness of the nonlinear solve is a topic of future investigation.

\subsection{Linear solvers}

Finding the solution of the nonlinear system (Eq. 25) using one or several Newton iterations requires solving the linear system (Eq. 28) for the iteration update. Since the HEVI splittings treat all the horizontal terms explicitly, Eq. (28) does not contain any coupling between the degrees of freedom in different vertical columns of the atmosphere. That is, the coupling introduced by the implicit terms only acts in the vertical direction, and the linear solve is therefore decomposable into a series of independent column-wise solves. The linear solves in each column are performed with the direct banded solver dgbsv from the Linear Algebra PACKage (LAPACK) (Anderson et al., 1999) without any need for interprocessor communication since the domain is partitioned by vertical columns across parallel processes. Moreover, when combined with the Rosenbrock-like approach, no communication is necessary in the nonlinear solve, and neither a nonlinear nor a linear tolerance needs to be set.

The inclusion of horizontal dynamics in the implicit function introduces coupling between degrees of freedom located in different columns, and a linear solve over the full domain is necessary to compute the Newton update. In this case, we employ a Newton-Krylov approach for the nonlinear solve where an approximate solution of Eq. (28) is found using the generalized minimal residual (GMRES) method (Saad and Schultz, 1986). Krylov methods require only the action of a matrix on a vector, and this operation is approximated through a finite difference computation:

$\mathbf{A}\left(z_{i}^{(m)}\right) \boldsymbol{v} \approx \frac{\boldsymbol{G}\left(z_{i}^{(m)}+\sigma \boldsymbol{v}\right)-\boldsymbol{G}\left(z_{i}^{(m)}\right)}{\sigma}$

where the increment $\sigma=1 /\|\boldsymbol{v}\|$ to ensure $\|\sigma \boldsymbol{v}\|=1$. Hence, constructing the full Jacobian matrix is unnecessary. We additionally precondition the GMRES solver on the right, using the HEVI-based column-wise direct solves described above. Since the HEVI methods treat only vertical dynamics implicitly, the horizontal dynamics in the IMEX splittings remain unpreconditioned. As GMRES is iterative, we consider the linear solution to be converged when the preconditioned 
residual vector $\boldsymbol{r}$ satisfies

$\|\boldsymbol{r}\| \leq 0.1 \epsilon_{\mathrm{L}} \epsilon$

where $\|\cdot\|$ is the WRMS norm. Like with the nonlinear solver error, the error in the linear solve must also be controlled to not interfere with the integration error; we therefore utilize the ARKode default value of $\epsilon_{\mathrm{L}}=0.05$.

\section{Numerical results}

We evaluate the accuracy and computational efficiency of the various implicit-explicit splittings, ARK methods, and solver options on two test cases. Section 5.1 presents results for the propagation of gravity waves on a sphere on a reduced-radius planet, and Sect. 5.2 focuses on the development of a baroclinic wave. Simulations are performed on the Cab Linux computing cluster at Lawrence Livermore National Laboratory. Each Cab node consists of two Intel Xeon eight-core Sandy Bridge processors with $32 \mathrm{~GB}$ of memory per node. All tests are run on six compute nodes using 96 MPI tasks. The absolute and relative tolerances in the numerical experiments are $\epsilon_{\mathrm{a}}=\epsilon_{\mathrm{r}}=10^{-4}$. These tolerances were chosen to produce results with ARKode that matched the solutions obtained with the native Tempest implementation of ARS232. The maximum number of Newton iterations is set to 10 , and the maximum number of GMRES iterations is set to 50, although these maximum values were only attained in one combination of splitting and solver as noted below.

\subsection{Gravity wave}

The gravity wave test as defined in Ullrich et al. (2012) begins with an initially balanced atmosphere on a reducedradius Earth (1/125 in size). A small potential temperature perturbation is added to the initial state causing the development of gravity waves. The domain is discretized using 2400 elements and 10 vertical levels. The test is simulated for $1 \mathrm{~h}$ with time steps of $0.01,0.1,0.5,1,2,4$, and $8 \mathrm{~s}$ with each of the different splittings, methods, and solvers described above. To compare the accuracy and efficiency of the different options, the root mean square error (RMSE) of the state vector with respect to a reference solution is computed at the final time. The reference solution is computed using a step size of $0.001 \mathrm{~s}$ with a fully explicit third-order five-stage Runge-Kutta method (KGU35) derived by Ullrich and implemented in Tempest (Guerra and Ullrich, 2016). This particular explicit method was created using the stability optimization presented in Kinnmark and Gray (1984) to maximize the stability region along the imaginary axis.

Accuracy and efficiency plots are shown in Figs. 1-6 for the gravity wave test. With the exception of the IMEX-B splitting, as noted below, the choice between a Rosenbrocklike approach or a full Newton iteration to solve the stage systems does not impact the maximum stable time step size of a given splitting or method, and both solver approaches produce nearly identical errors for this test case. Thus, using only a single Newton iteration provides a sufficiently accurate solution to the nonlinear stage systems in each time step. The Newton solver also consistently increases computational cost by approximately 20 to $50 \%$ over the Rosenbrock-like results with HEVI splittings and adds an additional cost of at least $10 \%$ with the IMEX options. Since there is not a significant benefit from a Newton solver in this test case, Figs. 1-6 focus only on results with a Rosenbrock-like approach. The choice of nonlinear solver is more important in the baroclinic wave test case and will be discussed further in the following section. Additionally, treating the vertical velocity or thermodynamic advection explicitly has a negligible impact on the solution error and integrator efficiency, so results with HEVI-B, C, and D are indistinguishable from those of HEVIA. As such, Figs. 1-6 present only HEVI-A, IMEX-A, and IMEX-B results, and any conclusions on the behavior or performance of HEVI-A also apply to HEVI-B, C, and D.

The second-order ARK methods can be divided into two groups based on accuracy regardless of the splitting choice. The more accurate group of methods consists of the lpm1, lpm2, lpum, and lspum optimized variants of SSP2(332) from Higueras et al. (2014), and the remaining second-order schemes comprise the second group with slightly less accuracy.

The approximate largest stable step size is consistent across the HEVI splittings. The ARK232, ARS232, and SSP3(332) methods are stable with $h_{n}=2 \mathrm{~s}$, the SSP2(332) methods are stable with $h_{n}=1 \mathrm{~s}$, and the remaining two methods are stable with $h_{n}=0.5 \mathrm{~s}$. With the IMEX-A option, all of the methods are able to achieve a step size of $2 \mathrm{~s}$, and including more implicit terms in IMEX-B increases the maximum step size to $8 \mathrm{~s}$ for all of the methods with the Rosenbrock-like approach. The IMEX-B splitting is the only case where integrator behavior differs when using the Newton solver rather than the Rosenbrock-like approach. In this instance, the maximum stable time step is smaller, approximately one-fourth the step size or smaller depending on the method, with the Newton iteration as it is unable to converge to the given tolerance with larger step sizes. Such behavior may be due to using the trivial predictor, and more sophisticated approaches could improve convergence with the Newton solver. Given the high cost of the IMEX-B splitting with Rosenbrock-like approach compared to the HEVI methods, the evaluation of alternative predictors with the Newton solver is left to future work.

The relative efficiency of the different ARK methods is also consistent across the splitting options. Despite requiring three implicit solves per time step, the optimized SSP2(332) methods from Higueras et al. (2014) are the most computationally efficient second-order approaches when higher solution accuracy is desired. Because of the larger maximum stable step size, ARK232, ARS232, and SSP3(332) provide slightly faster solution times with the HEVI splittings but 


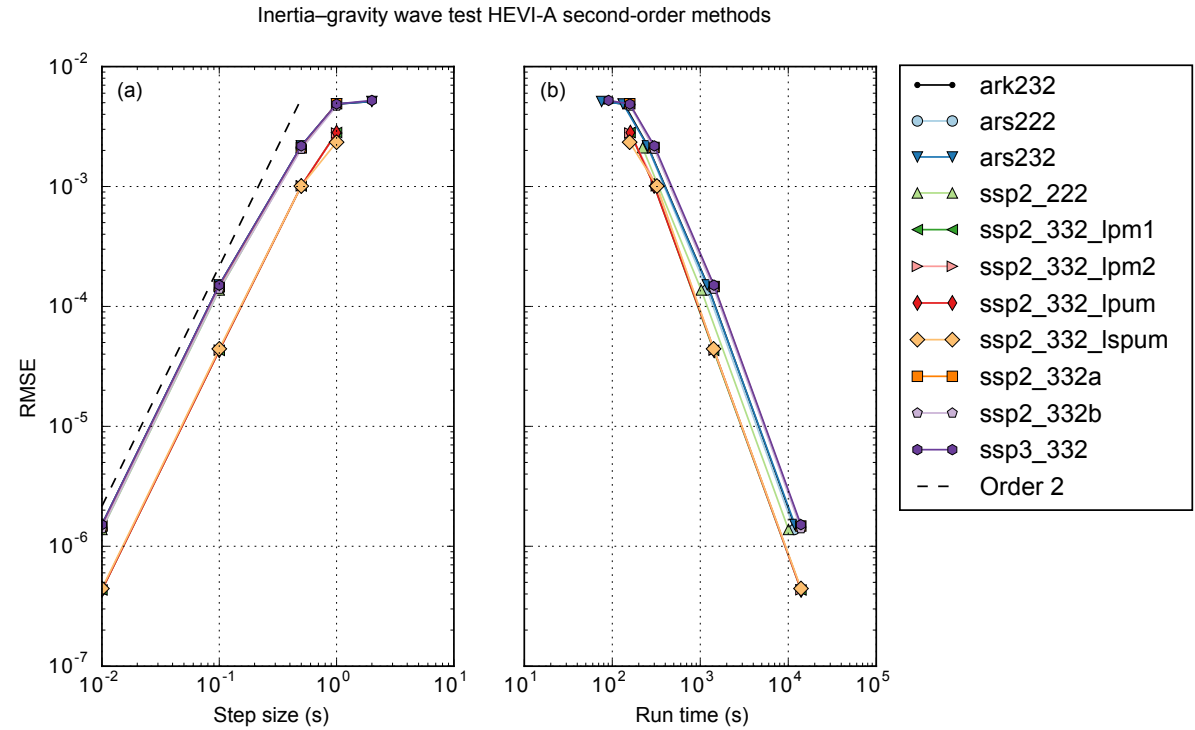

Figure 1. Accuracy (a) and efficiency (b) for the second-order methods using the Rosenbrock-like approach with the HEVI-A splitting. The dashed line represents second-order convergence. The ARK methods fall into two groups with similar accuracy. Results using multiple Newton iterations to compute the stage solutions give nearly identical error values to the Rosenbrock-like results but increase the computational cost by $20-50 \%$.
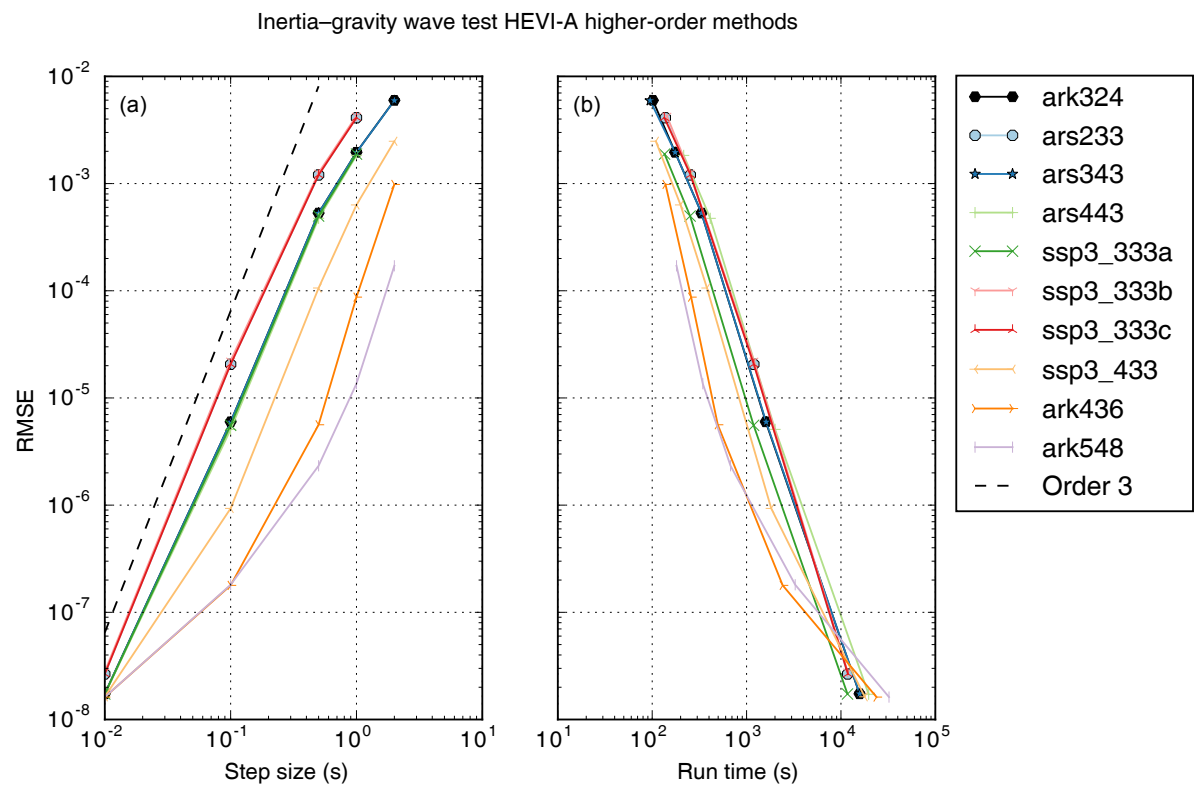

Figure 2. Accuracy (a) and efficiency (b) for the third-, fourth-, and fifth-order methods using the Rosenbrock-like approach with the HEVIA splitting. The dashed line represents third-order convergence. Results using multiple Newton iterations to compute the stage solutions give nearly identical error values to the Rosenbrock-like results but increase the computational cost by $20-50 \%$.

with larger error values. Because of the increased stability in the IMEX-A and IMEX-B tests, many of the second-order methods become competitive with the Higueras et al. (2014) SSP2(332) methods at $h_{n}=2 \mathrm{~s}$, but as with the HEVI splittings the optimized SSP2(332) methods are more efficient when greater accuracy is required. Making more terms explicit in the non-hydrostatic equations does not cause a sig- nificant difference in run times between the HEVI options. The inclusion of horizontally implicit terms and the additional communication necessary in each implicit solve with the IMEX-A and IMEX-B options increases the simulation time by approximately $25-60 \%$ over the HEVI results.

Across the splitting options, the majority of the thirdorder ARK methods produce solutions with approximately 

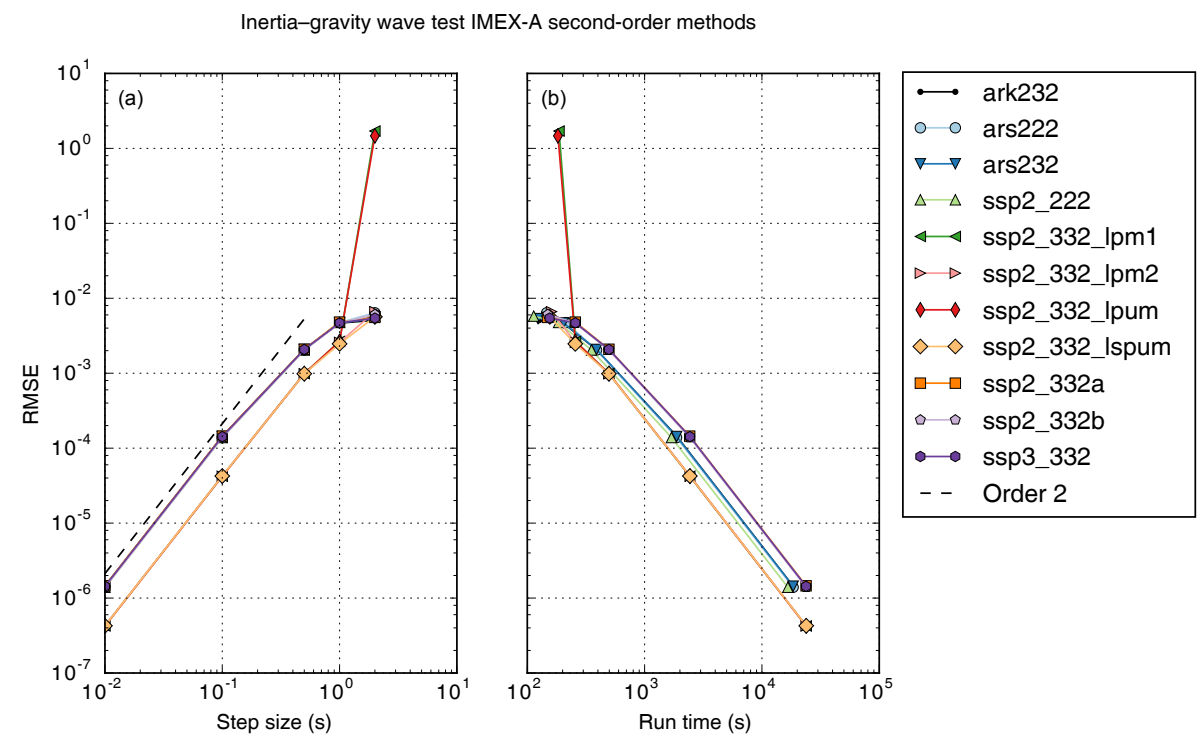

Figure 3. Accuracy (a) and efficiency (b) for the second-order methods using the Rosenbrock-like approach with the IMEX-A splitting. The ARK methods fall into two groups with similar accuracy. The dashed line represents second-order convergence. Results using multiple Newton iterations to compute the stage solutions give nearly identical error values to the Rosenbrock-like results but increase the computational cost by at least $10 \%$.
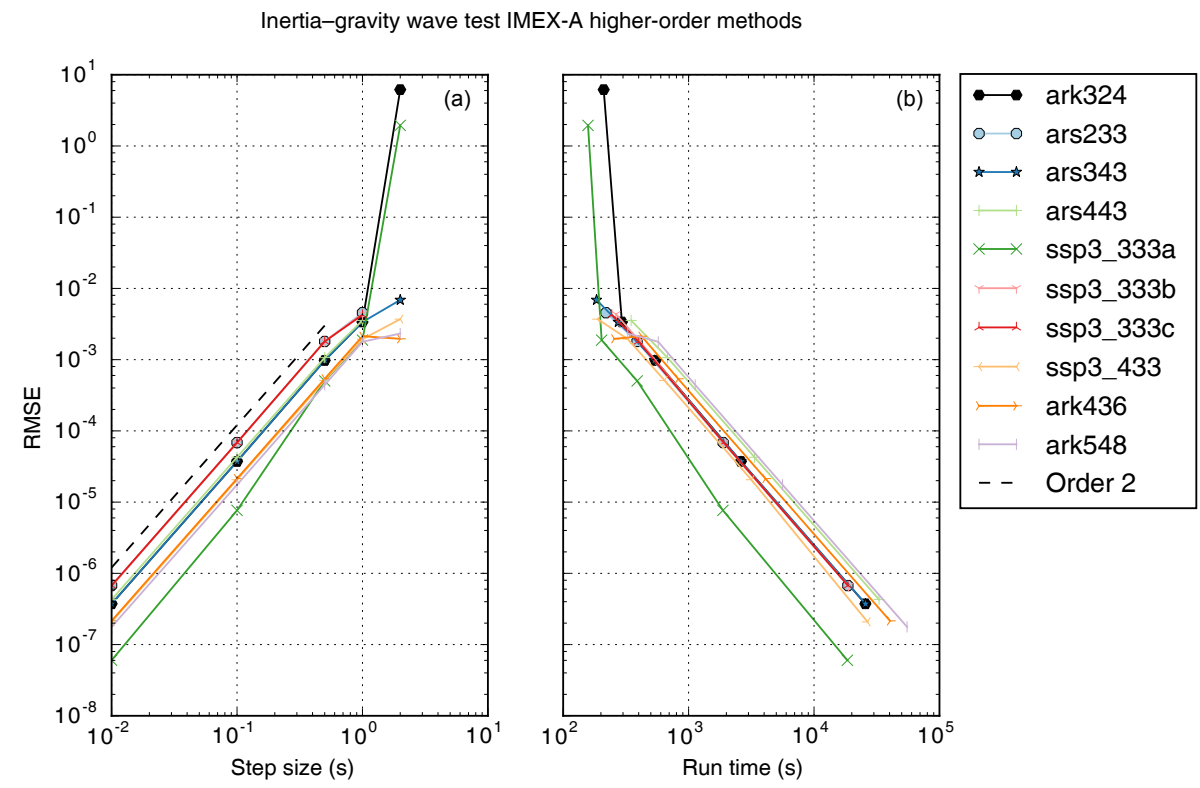

Figure 4. Accuracy (a) and efficiency (b) for the third-, fourth-, and fifth-order methods using the Rosenbrock-like approach with the IMEXA splitting. The dashed line represents second-order convergence. Results using multiple Newton iterations to compute the stage solutions give nearly identical error values to the Rosenbrock-like results but increase the computational cost by at least $10 \%$.

the same level of accuracy, with the exception of SSP3(433), which is generally more accurate, and ARS233, SSP3(333)b, and SSP3(333)c, which are less accurate. The fourth-order accurate ARK436 has smaller errors than all second- and third-order methods, and the fifth-order ARK548 method generally has the lowest error overall. The fifth-order ARK548 does not achieve the expected convergence rate, and with the IMEX-A splitting all of the methods drop to second-order convergence. Since the IMEX-B and HEVI-A methods show no such deterioration in accuracy, and they match IMEX-A but have more/fewer implicit terms, respectively, we believe that IMEX-A suffers from order reduction in the coupling terms. Specifically, it is likely that IMEX-A splits two large and opposite terms into explicit and implicit 


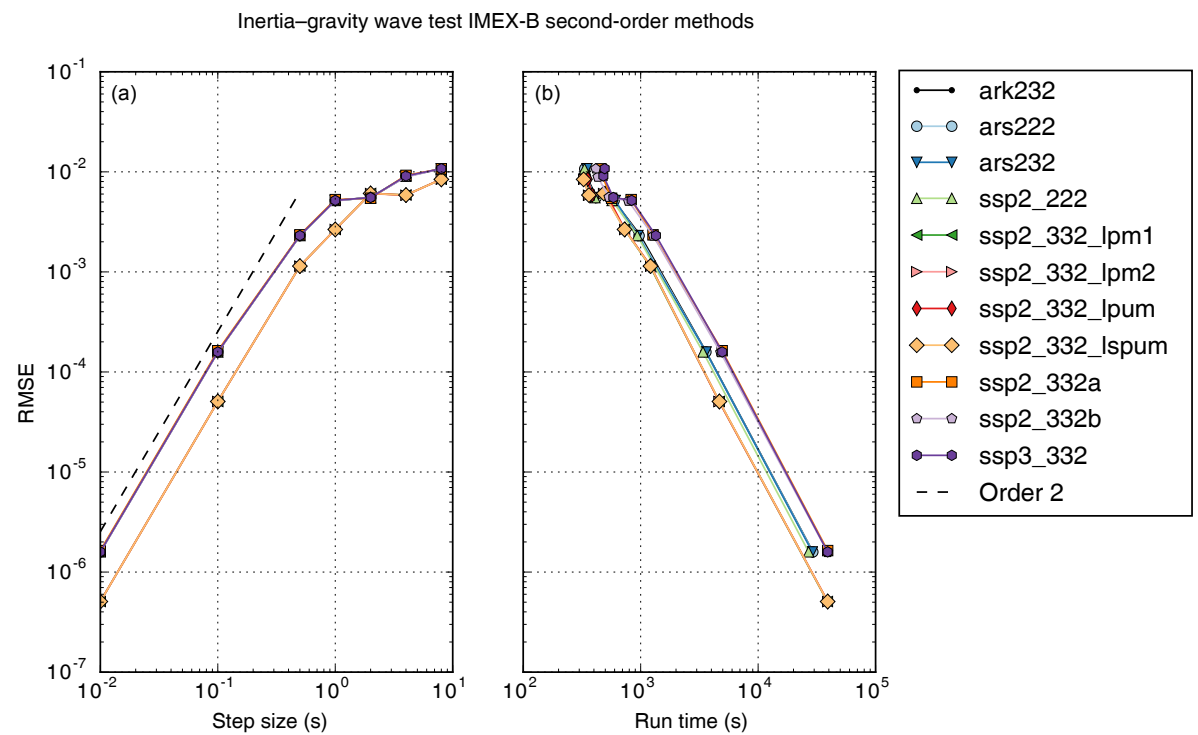

Figure 5. Accuracy (a) and efficiency (b) for the second-order methods using the Rosenbrock-like approach with the IMEX-B splitting. The ARK methods fall into two groups with similar accuracy. The dashed line represents second-order convergence. Results using multiple Newton iterations to compute the stage solutions give nearly identical error values to the Rosenbrock-like results but increase the computational cost by at least $10 \%$.
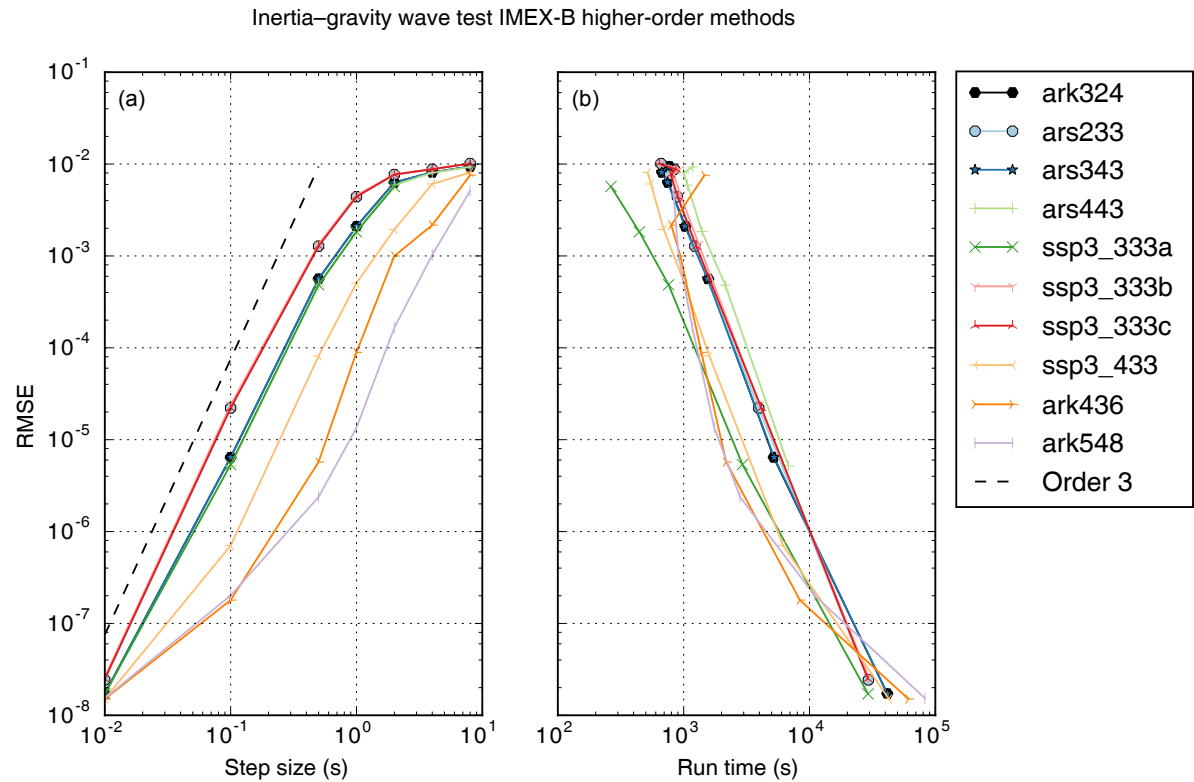

Figure 6. Accuracy (a) and efficiency (b) for the third-, fourth-, and fifth-order methods using the Rosenbrock-like approach with the IMEXB splitting. The dashed line represents third-order convergence. Results using multiple Newton iterations to compute the stage solutions give nearly identical error values to the Rosenbrock-like results but increase the computational cost by at least $10 \%$.

components, whereas IMEX-B and HEVI-A treat both terms consistently. As a result, partial derivatives of $f^{\mathrm{E}}$ and $f^{\mathrm{I}}$ in the IMEX-A splitting may have large magnitudes, resulting in increased stiffness, causing the order reduction.

Like the second-order methods, the choice of HEVI splitting does not effect the approximate maximum step size of a given third-order method. ARS233, ARS443, and
SSP3(333)a, b, and c all have a maximum steps size of $1 \mathrm{~s}$, and ARS343, SSP3(433), ARK324, ARK436, and ARK548 allow steps of up to $2 \mathrm{~s}$. SSP3(333)a is the only method to show a doubling in the maximum step size, going from 1 to $2 \mathrm{~s}$, due to the additional implicitness in IMEX-A. In the IMEX-B tests, all of the methods, with the exception of SSP3(333)a which does not gain stability, have an increase 
Table 1. Approximate largest stable step size (in seconds) for a 30-day run of the baroclinic wave test. Second-order methods are shown in the top section of the table and higher-order methods in the bottom section. For entries separated by " $/$ ", the left value is the step size for the Rosenbrock-like approach and the right value is the step size for the Newton solver. When a single step size is given, the Rosenbrock-like and Newton solvers were stable at the same step size. As in the gravity wave tests, the choice of a Rosenbrock-like or Newton solver does not generally impact the maximum stable step size, except in the case of IMEX-B which fails to converge. While the methods are able to complete a 30-day run at the step sizes listed below, the solutions produced are not sufficiently accurate in all cases and depend on the solver choice. Table 2 shows the approximate largest step sizes that give acceptable solutions.

\begin{tabular}{|c|c|c|c|c|c|c|}
\hline Method & HEVI-A & HEVI-B & HEVI-C & HEVI-D & IMEX-A & IMEX-B \\
\hline ARK232 & 200 & 200 & 135 & 135 & 480 & 480 \\
\hline ARS222 & 160 & 160 & 100/-* & $100 /-^{*}$ & 360 & 360 \\
\hline ARS232 & 200 & 200 & 135 & 135 & 480 & 540 \\
\hline SSP2(222) & 160 & 160 & 160 & 160 & 360 & 540 \\
\hline SSP2(332)lpm1 & 225 & 225 & $-^{*}$ & $-*$ & 400 & 540 \\
\hline SSP2(332)lpm2 & 225 & 225 & $-^{*}$ & - $^{*}$ & 400 & 540 \\
\hline SSP2(332)lpum & 225 & 225 & $-^{*}$ & $-*$ & 400 & 540 \\
\hline SSP2(332)lspum & 300 & 300 & $108 / 100$ & $108 / 100$ & 450 & 540 \\
\hline SSP2(332)a & 225 & 225 & 225 & 225 & 400 & 540 \\
\hline SSP2(332)b & 225 & 225 & 225 & 225 & 400 & 540 \\
\hline SSP3(332) & 320 & 320 & 320 & 320 & 540 & 540 \\
\hline ARK324 & 400 & 400 & 400 & 400 & 300 & 540 \\
\hline ARS233 & $-* / 320$ & $-* / 320$ & -* $^{*}$ & -* & -*/180 & -* \\
\hline ARS343 & 450 & 450 & 384 & 384 & 320 & 540 \\
\hline ARS443 & 300 & 300 & 300 & 300 & 320 & 540 \\
\hline SSP3(333)a & -* $^{*}$ & - $^{*}$ & $-^{*}$ & -* & -* $^{*}$ & -* \\
\hline SSP3(333)b & $-* / 320$ & $-* / 320$ & $-^{*}$ & $-^{*}$ & $-* / 180$ & -* \\
\hline SSP3(333)c & -*/320 & -*/320 & $-^{*}$ & $-^{*}$ & -*/180 & -* \\
\hline SSP3(433) & $200 / 216$ & $200 / 216$ & $135 / 150$ & $135 / 150$ & 480 & 540 \\
\hline ARK436 & 400 & 400 & -* $^{*}$ & -* & 450 & 540 \\
\hline ARK548 & 300 & 300 & $-^{*}$ & -* $^{*}$ & $432 / 450$ & 432 \\
\hline
\end{tabular}

* The method was not stable for 30 days with $h_{n} \geq 100 \mathrm{~s}$.

in maximum step size to $8 \mathrm{~s}$. As with second-order methods, the IMEX-B splitting is the only option where the choice of a Rosenbrock-like approach alters the integrator results by reducing the maximum step size due to lack of solver convergence.

Among the third-order methods, SSP3(443) is the most efficient method, except at the smallest step sizes where convergence begins to slow, and SSP3(333)a becomes faster for the same accuracy. Likewise, the fourth- and fifth-order methods are more cost effective until the convergence slows at the smallest time step sizes. SSP3(333)a is the best approach for lower accuracy levels in IMEX-B and is the best scheme in IMEX-A. For higher accuracy with IMEX-B, the faster convergence of ARK436 and ARK548 makes these approaches more efficient until convergence begins to slow at small step sizes. As with the second-order methods, HEVI$\mathrm{B}, \mathrm{C}$, and D do not present an advantage over HEVI-A in run time, and the additional communication required by the horizontal terms in the implicit portion of the IMEX methods is not offset by sufficient gains in step size.

With both the second- and higher-order integration methods, HEVI-A with the Rosenbrock-like approach is the best combination in this test case. For the most part, third-order methods outperform the second-order methods in terms of accuracy at a given step size. Since the third-order methods do not increase the maximum stable step size over that achieved by the second-order methods, the second-order schemes are more efficient at looser error requirements, and higher-order methods are best when more accuracy is necessary.

\subsection{Baroclinic wave}

The second test case simulates the development of a baroclinic wave over the course of approximately 10 days as described in Ullrich et al. (2014). For this test case, we focus on how the methods, splittings, and solvers perform near the maximum stable time step size in a 30-day simulation. The domain is discretized with 2400 elements and 30 vertical levels. Starting from a step size of $100 \mathrm{~s}, h_{n}$ is increased, using steps that evenly divide 1 day, until the method is unable to simulate 30 days without a solver failure. Table 1 lists the approximate largest step sizes for each of the methods. As with the results in the gravity wave test, the choice of a Rosenbrock-like or Newton solver does not generally impact 


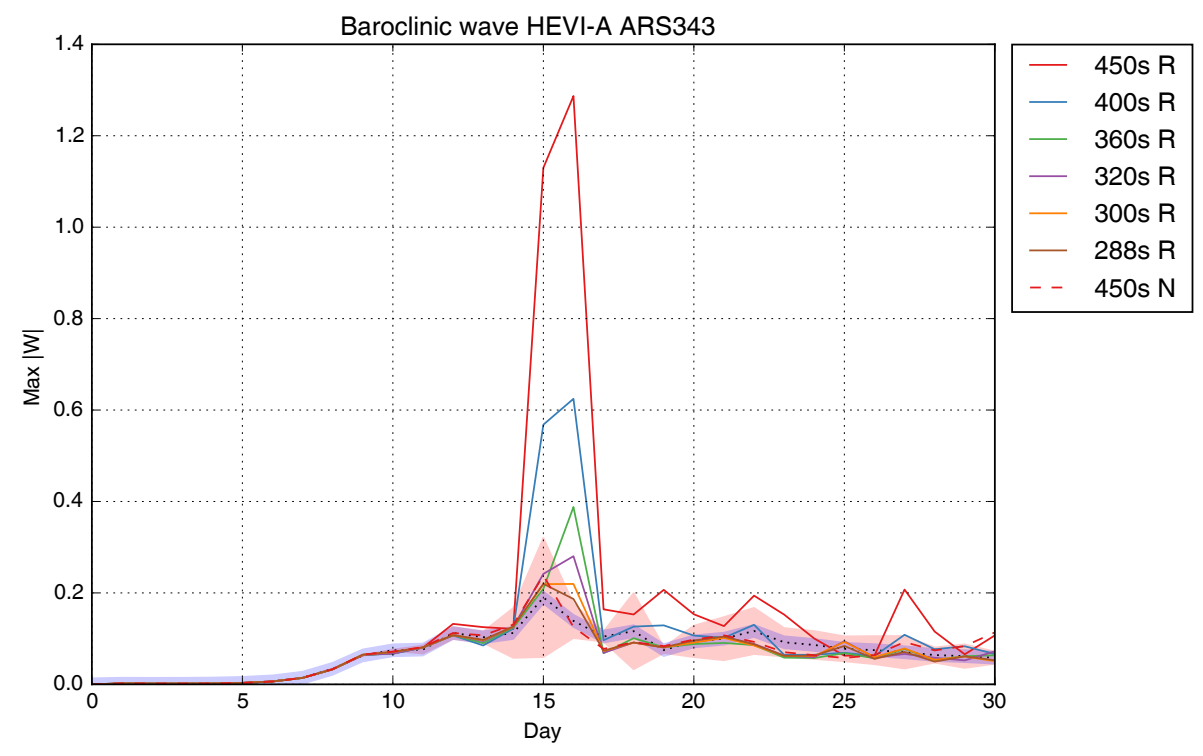

Figure 7. The maximum vertical velocity with ARS343 using the Rosenbrock-like approach (solid lines) and the Newton solver (dashed line) for various time step sizes. The light red region defines the $99 \%$ confidence interval from the explicit simulations with perturbed initial conditions, and the light purple region is $10 \%$ of the maximum deviation in the $99 \%$ confidence interval.

the largest stable step size for a given splitting or method with the exception of six methods (ARS222, SSP2(332)lspum, ARS233, SSP3(333)b, SSP3(333)c, and SSP3(433)). However, the solver selected does affect the quality of the solution produced at large time step sizes, and in many cases a smaller step size may be necessary to compute a sufficiently accurate solution with the Rosenbrock-like approach.

Since this problem produces a strong instability, comparisons against a highly resolved reference solution, as was used in the gravity wave test, do not yield a good metric for quality of a numerical solution. To define an acceptable numerical solution generated by the methods at any given time step, the results of the implicit-explicit simulations (HEVI or IMEX) are compared against the range of maximum vertical velocities produced by five explicit simulations with initial conditions perturbed by random noise. For a state variable $x$, the perturbed initial value is $x=x+\max (\kappa|x|, \kappa)$, where $\kappa$ is a normally distributed random number with mean 0 , standard deviation $\varepsilon \times 10^{11}$, and $\varepsilon$ is machine epsilon. The factor $10^{11}$ was selected to produce a max absolute difference (compared to the unperturbed explicit solution) in the vertical velocity after 1 day that is approximately an order of magnitude smaller than the maximum absolute difference observed with the ARS232 scheme using a step size of $200 \mathrm{~s}$. The explicit simulations utilize a Rayleigh sponge layer to damp problematic acoustic transients as, unlike with the ARK methods, there is not an implicit mechanism for dissipating these modes. The sponge layer is $8 \mathrm{~km}$ thick with a maximum strength of 0.5 and is applied after the Runge-Kutta (RK) update, by way of a backward Euler step, to relax all prognostic fields to the initial state continuously through the depth of the layer. The explicit simulations are advanced in time with the KGU35 method in Tempest using $h_{n}=2 \mathrm{~s}$ which is approximately the CFL step size for the simulation. The absolute maximum vertical velocity over the domain is computed at 1-day intervals for each test and a $99 \%$ confidence interval for the mean maximum vertical velocity is computed for each day using the $t$ distribution (Devore, 2008) to provide an upper bound on what is considered an acceptable solution. Figure 7 shows the $99 \%$ confidence interval for the maximum vertical velocity (the light red region) and the maximum vertical velocities for the HEVI-A splitting with ARS343 using various time step sizes. In the first few days of simulation, the velocities are slightly larger in the HEVI and IMEX formulations due to the presence of transients that are damped out by the presence of a Rayleigh sponge in our explicit simulations. Nonetheless, these transients are small and the vertical velocity is very similar to our reference range. The purple region is defined as $10 \%$ of the maximum deviation and the differences due to transients early in time fall within this region. To account for momentary large deviations from the confidence interval, the maximum vertical velocity of a method should fall in the reference range. The predictability of the solution breaks down over the last 15 days, and thus small, brief excursions outside of the reference range should not be considered anomalous. In the example in Fig. 7, ARS343 is stable with step sizes up to $450 \mathrm{~s}$. However, the results with the Rosenbrock-like approach (solid lines) produce exceptionally large vertical velocities that decrease with step size, and an acceptable solution is produced once the step size is below $300 \mathrm{~s}$. The solution using multiple Newton iterations (dashed line) is able to more accurately solve the nonlin- 
ear stage systems and yields an acceptable solution with a step size of $450 \mathrm{~s}$. The maximum acceptable time step sizes for the different splittings and integration methods using this methodology for defining an acceptable solution are given in Table 2. The corresponding normalized run times for the step sizes given in Table 2 are listed in Table 3.

Unlike the gravity wave test, treating the thermodynamic advection explicitly (HEVI-C and D) reduces the maximum stable and acceptable step size for some of the integration schemes. As a result, the increased number of time steps with HEVI-C and D can lead to longer run times than with HEVIA or B depending on the ARK method. Treating only the vertical velocity advection explicitly (HEVI-B) does not impact the maximum stable or acceptable step size, nor does it offer a significant advantage in run time over the HEVI-A setup. Handling more terms implicitly in IMEX-A and B can greatly increase the maximum stable step size but, in general, this does not translate into faster run times due to the increased solve cost and the smaller step sizes need to produce a sufficiently accurate solution. However, in a few cases with the Rosenbrock-like approach (ARK232, ARS222, ARS232, SSP2(332)lpm1, and SSP2(332)lpum), IMEX-A runs are faster than results with HEVI-C and D because of the larger acceptable time step size with the IMEX-A splitting and the minimal increase in solver cost due to the effectiveness of the vertical solve as a preconditioner (only two to four linear iterations are required per Newton iteration). The preconditioner is less effective in the IMEX-B splitting as more dynamics are included that are not treated by the preconditioner, so 16 to 25 linear iterations are needed per Newton iteration. As in the gravity wave test, the Newton solver does not perform well with the IMEX-B splitting and is unable to converge at step sizes for which the Rosenbrock-like approach gives an acceptable answer.

In this test, the increased accuracy and larger stability regions of the higher-order methods enable bigger time step sizes than the second-order methods with HEVI splittings and are somewhat less affected by the choice of HEVI splitting. The gains in step size are large enough to offset the third implicit stage solve required for ARK324 and ARS343, which consistently perform well. The ARS343 method is the fastest method across the HEVI splittings. ARS233, SSP3(333)b, and SSP3(333)c are less robust to the choice of splitting and solver but, when they produce an acceptable solution (HEVI-A and B with the Newton solver), are the second fastest methods, as they only require two implicit solves per step and have relatively large acceptable step size. ARS324 is more robust to the choice of spitting and solver, and is the third fastest method with HEVI-A and B, and the second fastest method with HEVI-C and D. The secondorder ARK232 and ARS232 methods give nearly identical performance and tie for fourth fastest method with the HEVI$\mathrm{A}$ and $\mathrm{B}$ splittings.

The ARK324 and ARS343 methods also highlight the potential advantage of the Newton solver over the Rosenbrock- like approach. With the exception of the second-order SSP methods (discussed below), the second-order methods studied produce acceptable solutions at their largest stable step size for HEVI splittings with either a Newton or Rosenbrocklike approach. As a result, there is no benefit from using the Newton solver for second-order methods and the Rosenbrock-like approach is always more efficient. At the larger stable step sizes enabled by higher-order methods, a Rosenbrock-like approach does not always give a sufficiently accurate answer, and a smaller step size is necessary to produce a good solution. Iterating to a converged stage value leads to better results at larger step sizes and, since only a few nonlinear iterations are necessary (on average two iterations per stage solve), a HEVI splitting with a Newton solver can outperform the Rosenbrock-like approach when the step size gain is sufficiently large.

While the other higher-order schemes are also able to take larger time steps than the second-order methods, they require more function evaluations or implicit solves than ARK324 or ARS343, and the step size gains are not enough to overcome the additional costs. Four of the third-order methods (ARS233, SSP3(333)b, and SSP3(333)c with the Rosenbrock-like solver, and SSP3(333)a with either solver) are not stable for 30 days at step sizes of at least $100 \mathrm{~s}$ with any of the splittings. These failures are likely because the implicit parts are not L stable (or even A stable for SSP3(333)a), and the fastest dynamics of the system are not sufficiently damped. These methods did perform well in the gravity wave test case, which might have been due to the reduced domain size altering the eigenvalues of the system.

However, L stability does not guarantee that a method will produce a good solution. All of the SSP methods tested, with the exception of SSP3(333)a, b, and c, are L stable but only SSP2(332)a consistently gives acceptable results with the HEVI splittings. The other SSP methods are generally stable but give vertical velocities an order of magnitude larger than the mean solution value with step sizes above 100 s. Exceptions to this behavior include SSP2(332)b, which underestimates the vertical velocities, and SSP3(333)b and c, which have acceptable solutions at their maximum stable step size when using the Newton solver with the HEVI-A or B splittings. The better performance of SSP3(333)b and c may be attributable to having the same $c$ values for both the implicit and explicit methods, as they are the only SSP methods tested with this property. While having identical $\boldsymbol{c}$ values is not necessary for producing acceptable solutions (e.g., SSP2(332)a), having the stage values aligned in time appears beneficial. Acceptable solutions with the other SSP methods are produced with the IMEX-A and B splittings, suggesting that the inaccuracy with SSP methods may be related to the splitting error in the schemes. Comparing with the gravity wave results where the SSP methods were both accurate and efficient suggests that again the reduced domain size may have played a role in the quality of the results by altering the eigenvalues of the system, since the stability region of the explicit por- 
Table 2. Approximate largest step size (in seconds) for a 30-day run of the baroclinic wave test that produces acceptable maximum vertical velocities over time. Second-order methods are shown in the top section of the table and higher-order methods in the bottom section. For entries separated by "/", the left value is the step size for the Rosenbrock-like approach and the right value is the step size for the Newton solver. When a single step size is given, the Rosenbrock-like and Newton solvers gave acceptable solutions at the same step size.

\begin{tabular}{|c|c|c|c|c|c|c|}
\hline Method & HEVI-A & HEVI-B & HEVI-C & HEVI-D & IMEX-A & IMEX-B \\
\hline ARK232 & 200 & 200 & 128 & 128 & $320 / 480$ & 320 \\
\hline ARS222 & 160 & 160 & $100 /-^{\mathrm{a}}$ & $100 /-^{\mathrm{a}}$ & 360 & 320 \\
\hline ARS232 & 200 & 200 & 128 & 128 & $360 / 480$ & 360 \\
\hline SSP2(222) & $-b$ & ${ }^{b}$ & $-b$ & $-b$ & $-b$ & 360 \\
\hline SSP2(332)lpm1 & $-\mathrm{b}$ & $-\mathrm{b}$ & $-{ }^{\mathrm{a}}$ & $-^{\mathrm{a}}$ & $400 / 200$ & 400 \\
\hline SSP2(332)lpm2 & $-\mathrm{b}$ & $-b$ & $-^{\mathrm{a}}$ & $-^{\mathrm{a}}$ & $100 / 150$ & 450 \\
\hline SSP2(332)lpum & $-\mathrm{b}$ & $-b$ & $-^{\mathrm{a}}$ & $-^{\mathrm{a}}$ & $360 / 108$ & 450 \\
\hline SSP2(332)lspum & $-\mathrm{b}$ & $-\mathrm{b}$ & $-\mathrm{b}$ & $-\mathrm{b}$ & $120 / 450$ & 450 \\
\hline SSP2(332)a & 225 & 225 & 225 & 225 & $300 / 400$ & 320 \\
\hline SSP2(332)b & $-\mathrm{b}$ & $-b$ & $-b$ & $-b$ & $270 / 400$ & 320 \\
\hline SSP3(332) & $-\mathrm{b}$ & $-\mathrm{b}$ & $-\mathrm{b}$ & $-\mathrm{b}$ & $-\mathrm{b}$ & 384 \\
\hline ARK324 & $300 / 400$ & $300 / 400$ & $300 / 400$ & $300 / 400$ & 300 & 320 \\
\hline ARS233 & $-{ }^{a} / 320$ & $-{ }^{\mathrm{a}} / 320$ & $-{ }^{\mathrm{a}}$ & $-{ }^{\mathrm{a}}$ & $-{ }^{\mathrm{a}} / 180$ & $-^{\mathrm{a}}$ \\
\hline ARS343 & $288 / 450$ & $300 / 450$ & $300 / 384$ & $300 / 384$ & $300 / 320$ & 300 \\
\hline ARS443 & 300 & 300 & $270 / 300$ & $270 / 300$ & $300 / 320$ & 320 \\
\hline SSP3(333)a & $-{ }^{\mathrm{a}}$ & $-{ }^{\mathrm{a}}$ & $-^{\mathrm{a}}$ & $-^{\mathrm{a}}$ & $-^{\mathrm{a}}$ & $-^{\mathrm{a}}$ \\
\hline SSP3(333)b & $-{ }^{\mathrm{a}} / 320$ & $-{ }^{\mathrm{a}} / 320$ & $-{ }^{\mathrm{a}}$ & $-{ }^{\mathrm{a}}$ & $-{ }^{\mathrm{a}} / 180$ & $-^{\mathrm{a}}$ \\
\hline SSP3(333)c & $-{ }^{\mathrm{a}} / 320$ & $-{ }^{\mathrm{a}} / 320$ & $-{ }^{\mathrm{a}}$ & $-{ }^{\mathrm{a}}$ & $-{ }^{\mathrm{a}} / 180$ & $-^{\mathrm{a}}$ \\
\hline SSP3(433) & $-^{\mathrm{b}}$ & $-b$ & $-\mathrm{b}$ & $-^{b}$ & 480 & 480 \\
\hline ARK436 & 400 & 400 & $-{ }^{\mathrm{a}}$ & $-{ }^{\mathrm{a}}$ & $384 / 450$ & 384 \\
\hline ARK548 & 300 & 300 & $-{ }^{\mathrm{a}}$ & $-{ }^{\mathrm{a}}$ & $400 / 450$ & 384 \\
\hline
\end{tabular}

a The method was not stable for 30 days with $h_{n} \geq 100 \mathrm{~s} .{ }^{\mathrm{b}}$ The method was unable to produce an acceptable solution with $h_{n} \geq 100 \mathrm{~s}$.

tion of many of the SSP methods does not include part of the imaginary axis.

\section{Conclusions}

Considering the results of the gravity wave and baroclinic wave test cases, the HEVI-A and B approaches are the most accurate and efficient of the implicit-explicit splittings considered. Treating some of the vertical dynamics of HEVIA explicitly does not provide a noticeable gain in efficiency from simpler implicit systems and, in the case of HEVI-C and $\mathrm{D}$, can lead to reduced step sizes in the baroclinic wave test. Adding horizontally implicit terms to the HEVI-A formulation does increase the maximum stable step size, but the gains are not large enough to overcome the added cost of a globally implicit solve.

While SSP methods are the most accurate and efficient approaches in the gravity wave test case, they generally do not preform well in the baroclinic wave test (with some notable exceptions), possibly due to error from the choice of implicit-explicit splitting. The reduced domain size seemed to skew the gravity wave test results in favor of these methods while the other ARK schemes perform well in both test cases. Additionally, the gravity wave test case does not show a benefit, in terms of maximum stable step size, with higherorder methods, although it does highlight their greater efficiency when higher accuracy is required. Again, these results are likely due to the reduced domain size altering the eigenvalues of the system. In the baroclinic wave test on a full-size Earth, higher-order methods produce accurate solutions at step sizes large enough to have faster run times than second-order schemes involving fewer implicit solves.

At the larger time step sizes enabled by higher-order methods in the baroclinic wave tests, the choice of nonlinear solver approach becomes an important consideration. A Rosenbrock-like approach limits the cost associated with multiple Newton iterations but may require a reduced step size to obtain an accurate solution. Taking larger steps is possible by iterating stage solutions to convergence with Newton's method. The additional cost is minimal and can be offset by the larger step size. The choice of predictor values was not considered in this work but could lead to more efficient nonlinear solves with Newton's method or more accurate Rosenbrock-like schemes.

The HEVI-A and B configurations produced nearly identical results, while the HEVI-C and D options were problematic for some methods in the baroclinic wave test. Since HEVI-A employs the same discretization for kinetic energy 
Table 3. The corresponding run times for the approximate largest acceptable step sizes in Table 2. Second-order methods are shown in the top section of the table and higher-order methods in the bottom section. The times have been normalized by the fastest simulation time, HEVI-B using ARS343 with the Newton solver (1372.483 s). The value on the left of the "/" divider is the time for the Rosenbrock-like approach and the value on the right is the time for the Newton solver.

\begin{tabular}{|c|c|c|c|c|c|c|}
\hline Method & HEVI-A & HEVI-B & HEVI-C & HEVI-D & IMEX-A & IMEX-B \\
\hline ARK232 & $1.19 / 1.61$ & $1.19 / 1.61$ & $1.81 / 2.47$ & $1.82 / 2.43$ & $1.77 / 2.04$ & 6.84 \\
\hline ARS222 & $1.48 / 2.01$ & $1.48 / 2.02$ & $2.30 /-^{\mathrm{a}}$ & $2.31 /-^{\mathrm{a}}$ & $1.56 / 2.31$ & 6.67 \\
\hline ARS232 & $1.19 / 1.61$ & $1.20 / 1.60$ & $1.83 / 2.46$ & $1.81 / 2.43$ & $1.53 / 2.02$ & 6.49 \\
\hline SSP2(222) & $-\mathrm{b}$ & $-^{b}$ & $-b$ & $-^{\mathrm{b}}$ & $-b$ & 6.47 \\
\hline SSP2(332)lpm1 & $-\mathrm{b}$ & $-b$ & $-^{\mathrm{a}}$ & $-^{\mathrm{a}}$ & $1.74 / 4.76$ & 6.86 \\
\hline SSP2(332)lpm2 & $-\mathrm{b}$ & $-b$ & $-^{\mathrm{a}}$ & $-^{\mathrm{a}}$ & $5.44 / 6.01$ & 6.62 \\
\hline SSP2(332)lpum & $-\mathrm{b}$ & $-b$ & $-b$ & $-b$ & $1.89 / 7.35$ & 6.60 \\
\hline SSP2(332)lspum & $-\mathrm{b}$ & $-\mathrm{b}$ & $-\mathrm{b}$ & $-\mathrm{b}$ & $4.79 / 2.84$ & 6.69 \\
\hline SSP2(332)a & $1.27 / 1.83$ & $1.25 / 1.79$ & $1.24 / 1.92$ & $1.24 / 1.86$ & $2.29 / 3.02$ & 9.48 \\
\hline SSP2(332)b & $-{ }^{\mathrm{b}}$ & $-{ }^{\mathrm{b}}$ & $-{ }^{b}$ & $-{ }^{\mathrm{b}}$ & $2.50 / 3.01$ & 8.90 \\
\hline SSP3(332) & $-^{b}$ & $-{ }^{b}$ & $-{ }^{b}$ & $-{ }^{b}$ & $-\mathrm{b}$ & 9.36 \\
\hline ARK324 & $1.06 / 1.14$ & $1.07 / 1.13$ & $1.06 / 1.23$ & $1.05 / 1.21$ & $2.93 / 4.41$ & 13.28 \\
\hline ARS233 & $-{ }^{\mathrm{a}} / 1.03$ & $-{ }^{\mathrm{a}} / 1.02$ & $-^{\mathrm{a}}$ & $-^{\mathrm{a}}$ & $-{ }^{\mathrm{a}} / 4.38$ & $-^{\mathrm{a}}$ \\
\hline ARS343 & $1.10 / 1.02$ & $1.09 / 1.00$ & $1.07 / 1.21$ & $1.05 / 1.19$ & $2.86 / 4.08$ & 13.21 \\
\hline ARS443 & $1.34 / 1.90$ & $1.32 / 1.90$ & $1.46 / 1.88$ & $1.45 / 1.84$ & $3.82 / 5.48$ & 19.26 \\
\hline SSP3(333)a & $-^{\mathrm{a}}$ & $-^{\mathrm{a}}$ & $-^{\mathrm{a}}$ & $-^{\mathrm{a}}$ & $-^{\mathrm{a}}$ & $-^{\mathrm{a}}$ \\
\hline SSP3(333)b & $-{ }^{\mathrm{a}} / 1.04$ & $-{ }^{\mathrm{a}} / 1.02$ & $-^{\mathrm{a}}$ & $-^{\mathrm{a}}$ & $-{ }^{\mathrm{a}} / 4.50$ & $-^{\mathrm{a}}$ \\
\hline SSP3(333)c & $-{ }^{\mathrm{a}} / 1.03$ & $-{ }^{\mathrm{a}} / 1.02$ & $-^{\mathrm{a}}$ & $-^{\mathrm{a}}$ & $-{ }^{\mathrm{a}} / 4.64$ & $-^{\mathrm{a}}$ \\
\hline SSP3(433) & $-^{b}$ & $-{ }^{b}$ & $-{ }^{b}$ & $-{ }^{b}$ & $2.27 / 3.97$ & 10.19 \\
\hline ARK436 & $1.21 / 1.76$ & $1.20 / 1.74$ & $-^{\mathrm{a}}$ & $-^{\mathrm{a}}$ & $3.28 / 4.65$ & 14.14 \\
\hline ARK548 & $2.14 / 3.16$ & $2.15 / 3.11$ & $-^{\mathrm{a}}$ & $-^{\mathrm{a}}$ & $3.96 / 5.75$ & 17.31 \\
\hline
\end{tabular}

${ }^{\text {a }}$ The method was not stable for 30 days with $h_{n} \geq 100 \mathrm{~s}$. ${ }^{\mathrm{b}}$ The method was unable to produce an acceptable solution with $h_{n} \geq 100 \mathrm{~s}$.

transport as vertical mass transport without a significant difference in computational cost, it is preferred over the HEVIB option. Overall, the third-order ARS343 method shows excellent performance across the splitting and solver options. ARS233, SSP3(333)b, and SSP3(333)c are also efficient third-order methods but their performance depends on the appropriate choice of splitting and solver. A more robust runner-up method is the third-order ARK324 method which follows closely behind ARS343 in run times. The secondorder ARS232 and ARK232 methods highlighted in Weller et al. (2013) and Giraldo et al. (2013) using the Rosenbrocklike were also very efficient options.

The ARK324 and ARK232 are of particular interest as both include an embedded method which will be leveraged for future studies on temporal adaptivity in atmospheric simulations using ARKode. Varying the time step size can enable greater efficiency by placing temporal accuracy where is it needed most to capture dynamics of interest. Additionally, we plan on further evaluating the methods in this study on the 2016 dynamical core model intercomparison project (DCMIP2016) test cases to better understand the impacts of coupling with simplified physics on performance of implicitexplicit splittings and integration methods.
Code availability. Tempest is available through the Git repository at https://github.com/paullric/tempestmodel, and ARKode is available as part of the SUNDIALS library of solvers downloadable from http://computation.llnl.gov/projects/sundials. The version of Tempest that includes ARKode interfaces used for this work and splittings with horizontally implicit terms is available in the Git repository at https://github.com/gardner48/tempestmodel. The versions of Tempest with ARKode interfaces used in this work are archived at https://doi.org/10.5281/zenodo.1162309. 


\section{Appendix A: ARK method properties}

In Table A1, we provide a variety of theoretical properties of each of the ARK methods used in this paper. While we do not reproduce each Butcher table here, references for each method are provided in Sect. 3.2. For each method, we provide the following information:

- number of implicit solves per step ( $f^{\mathrm{I}}$ column $)$ - the number of nonzero entries on the diagonal of $\mathbf{A}^{\mathrm{I}}$;

- number of explicit stages ( $f^{\mathrm{E}}$ column $)$ - the total number of RK stages that involve calls to $f^{\mathrm{E}}$;

- order - theoretical order of accuracy of the explicit Runge-Kutta (ERK) method, the diagonally implicit Runge-Kutta (DIRK) method, and the overall ARK method (including coupling conditions);

- stage order - theoretical order of accuracy of stages (relevant for order reduction on stiff problems), again for the ERK stages, DIRK stages, and the overall ARK stages;

- stability - A, L, and B stability for the DIRK portion of each method;
- S.A. DIRK - if the DIRK method is stiffly accurate (i.e., the last row of $\mathbf{A}^{\mathbf{I}}$ is the same as the $\boldsymbol{b}^{\mathbf{I}}$ );

- S.A. ERK - if the ERK method is "stiffly accurate" (i.e., the last row of $\mathbf{A}^{\mathrm{E}}$ is the same as the $\boldsymbol{b}^{\mathrm{E}}$ );

- same solution weights (b column) - if the ERK and DIRK methods have the same weights to compute $\boldsymbol{y}_{n}$ (i.e., $\boldsymbol{b}^{\mathrm{E}}=\boldsymbol{b}^{\mathrm{I}}$ ) and will preserve linear invariants of the problem to machine precision;

- same abscissa ( $\boldsymbol{c}$ column) - if the stages in the ERK and DIRK methods are evaluated at the same stage times (i.e., $c^{\mathrm{E}}=\boldsymbol{c}^{\mathrm{I}}$ ); and

- maximum stable explicit step along the imaginary axis as this application has purely imaginary eigenvalues, we numerically compute the largest $y_{\max } \in \mathbb{R}$ such that the ERK portion of the method is stable for all $\lambda=i y, 0 \leq$ $y \leq y_{\max }$, using a bisection method with tolerance $10^{-6}$. If the method is analytically unstable for any nonzero values along the imaginary axis, we list " 0 ".

Table A1. Properties for each of the ARK methods used in this paper. The column headings are described in the above text.

\begin{tabular}{|c|c|c|c|c|c|c|c|c|c|c|c|c|c|c|c|c|}
\hline \multirow{2}{*}{ Method } & \multirow{2}{*}{$f^{\mathrm{I}}$} & \multirow{2}{*}{$f^{\mathrm{E}}$} & \multicolumn{3}{|c|}{ Order } & \multicolumn{3}{|c|}{ Stage order } & \multicolumn{3}{|c|}{ Stability } & \multirow{2}{*}{$\begin{array}{l}\text { S.A. } \\
\text { DIRK }\end{array}$} & \multirow{2}{*}{$\begin{array}{l}\text { S.A. } \\
\text { ERK }\end{array}$} & \multirow{2}{*}{$\boldsymbol{b}$} & \multirow{2}{*}{$c$} & \multirow{2}{*}{ Max exp } \\
\hline & & & $\mathrm{E}$ & I & A & $\mathrm{E}$ & I & A & $\mathrm{A}$ & $\mathrm{L}$ & B & & & & & \\
\hline ARK232 & 2 & 3 & 2 & 2 & 2 & 1 & 2 & 1 & $\checkmark$ & $\checkmark$ & $\mathrm{X}$ & $\checkmark$ & $\mathrm{X}$ & $\checkmark$ & $\checkmark$ & $\sim 1.73$ \\
\hline ARS222 & 2 & 3 & 2 & 2 & 2 & 1 & 1 & 1 & $\checkmark$ & $\checkmark$ & $X$ & $\checkmark$ & $\checkmark$ & $X$ & $\checkmark$ & 0 \\
\hline ARS232 & 2 & 3 & 2 & 2 & 2 & 1 & 1 & 1 & $\checkmark$ & $\checkmark$ & $\mathrm{X}$ & $\checkmark$ & X & $\checkmark$ & $\checkmark$ & $\sim 1.73$ \\
\hline SSP2(222) & 2 & 2 & 2 & 2 & 2 & 1 & 1 & 0 & $\checkmark$ & $\checkmark$ & $\checkmark$ & $X$ & $\mathrm{X}$ & $\checkmark$ & $\mathrm{X}$ & 0 \\
\hline SSP2(332)lpm1 & 3 & 3 & 2 & 2 & 2 & 1 & 1 & 0 & $\checkmark$ & $\checkmark$ & $\mathrm{X}$ & $X$ & $X$ & $\checkmark$ & $X$ & 0 \\
\hline SSP2(332)lpm2 & 3 & 3 & 2 & 2 & 2 & 1 & 1 & 0 & $\checkmark$ & $\checkmark$ & $X$ & $X$ & $X$ & $\checkmark$ & $X$ & 0 \\
\hline SSP2(332)lpum & 3 & 3 & 2 & 2 & 2 & 1 & 1 & 0 & $\checkmark$ & $\checkmark$ & $\mathrm{X}$ & $X$ & $X$ & $\checkmark$ & $X$ & 0 \\
\hline SSP2(332)lspum & 3 & 3 & 2 & 2 & 2 & 1 & 1 & 0 & $\checkmark$ & $\checkmark$ & $\mathrm{X}$ & $X$ & $X$ & $\checkmark$ & $X$ & $\sim 1.2$ \\
\hline SSP2(332)a & 3 & 3 & 2 & 2 & 2 & 1 & 1 & 0 & $\checkmark$ & $\checkmark$ & $\mathrm{X}$ & $\checkmark$ & $X$ & $\checkmark$ & $X$ & 0 \\
\hline SSP2(332)b & 3 & 3 & 2 & 2 & 2 & 1 & 1 & 0 & $\checkmark$ & $\checkmark$ & $\mathrm{X}$ & $\checkmark$ & $\mathrm{X}$ & $\checkmark$ & $\mathrm{X}$ & 0 \\
\hline SSP3(332) & 3 & 3 & 3 & 2 & 2 & 1 & 1 & 0 & $\checkmark$ & $\checkmark$ & $\mathrm{X}$ & $\mathrm{X}$ & $\mathrm{X}$ & $\checkmark$ & $\mathrm{X}$ & $\sim 1.73$ \\
\hline ARK324 & 3 & 4 & 3 & 3 & 3 & 1 & 2 & 1 & $\checkmark$ & $\checkmark$ & $\mathrm{X}$ & $\checkmark$ & $\mathrm{X}$ & $\checkmark$ & $\checkmark$ & $\sim 2.48$ \\
\hline ARS233 & 2 & 3 & 3 & 4 & 3 & 1 & 1 & 1 & $\checkmark$ & $X$ & $\checkmark$ & $X$ & $\mathrm{X}$ & $\checkmark$ & $\checkmark$ & $\sim 1.73$ \\
\hline ARS343 & 3 & 4 & 3 & 3 & 3 & 1 & 1 & 1 & $\checkmark$ & $\checkmark$ & $X$ & $\checkmark$ & $\mathrm{X}$ & $\checkmark$ & $\checkmark$ & $\sim 2.83$ \\
\hline ARS443 & 4 & 4 & 3 & 3 & 3 & 1 & 1 & 1 & $\checkmark$ & $\checkmark$ & $\mathrm{X}$ & $\checkmark$ & $\checkmark$ & $X$ & $\checkmark$ & $\sim 1.57$ \\
\hline SSP3(333)a & 2 & 3 & 3 & 3 & 3 & 1 & 1 & 1 & $X$ & $X$ & $X$ & $X$ & $X$ & $\checkmark$ & $X$ & $\sim 1.73$ \\
\hline SSP3(333)b & 2 & 3 & 3 & 3 & 3 & 1 & 1 & 1 & $\checkmark$ & $X$ & $X$ & $X$ & $X$ & $\checkmark$ & $\checkmark$ & $\sim 1.73$ \\
\hline SSP3(333)c & 2 & 3 & 3 & 3 & 3 & 1 & 1 & 1 & $\checkmark$ & $X$ & $\mathrm{X}$ & $X$ & $X$ & $\checkmark$ & $\checkmark$ & $\sim 1.73$ \\
\hline SSP3(433) & 4 & 3 & 3 & 3 & 3 & 1 & 1 & 0 & $\checkmark$ & $\checkmark$ & $\mathrm{X}$ & $X$ & $\mathrm{X}$ & $\checkmark$ & $\mathrm{X}$ & $\sim 1.73$ \\
\hline ARK436 & 5 & 6 & 4 & 4 & 4 & 1 & 2 & 1 & $\checkmark$ & $\checkmark$ & $X$ & $\checkmark$ & $X$ & $\checkmark$ & $\checkmark$ & $\sim 4.00$ \\
\hline ARK548 & 7 & 8 & 5 & 5 & 5 & 1 & 2 & 1 & $\checkmark$ & $\checkmark$ & $X$ & $\checkmark$ & $X$ & $\checkmark$ & $\checkmark$ & $\sim 0.79$ \\
\hline
\end{tabular}


Competing interests. The authors declare that they have no conflict of interest.

Acknowledgements. Support for this work was provided by the Department of Energy, Office of Science Scientific Discovery through Advanced Computing (SciDAC) project "A Non-hydrostatic Variable Resolution Atmospheric Model in ACME". This work was performed under the auspices of the US Department of Energy by Lawrence Livermore National Laboratory under contract DE-AC52-07NA27344. LLNL-JRNL-737448.

Edited by: Simone Marras

Reviewed by: two anonymous referees

\section{References}

Anderson, E., Bai, Z., Bischof, C., Blackford, S., Demmel, J., Dongarra, J., Du Croz, J., Greenbaum, A., Hammarling, S., McKenney, A., and Sorensen, D.: LAPACK Users' Guide, Society for Industrial and Applied Mathematics, 3rd Edn., Philadelphia, PA, 425 pp., 1999.

Ascher, U. M., Ruuth, S. J., and Spiteri, R. J.: Implicitexplicit Runge-Kutta methods for time-dependent partial differential equations, Appl. Numer. Math., 25, 151-167, https://doi.org/10.1016/S0168-9274(97)00056-1, 1997.

Conde, S., Gottlieb, S., Grant, Z. J., and Shadid, J. N.: Implicit and Implicit-Explicit Strong Stability Preserving Runge-Kutta Methods with High Linear Order, J. Sci. Comput., 73, 667-690, https://doi.org/10.1007/s10915-017-0560-2, 2017.

Devore, J. L.: Probability and Statistics for Engineering and the Sciences, 7th Edn., Thomson Brooks/Cole, 736 pp., 2008.

Gardner, D. J., Reynolds, D. R., Hamon, F. P., Woodward, C. S., Ullrich, P., Guerra, J. E., Lelbach, B. A., and Banide, A. V.: Tempest+ARKode IMEX Tests, https://doi.org/10.5281/zenodo.1162309, 2017.

Giraldo, F. X., Kelly, J. F., and Constantinescu, E. M.: ImplicitExplicit Formulations of a Three-Dimensional Nonhydrostatic Unified Model of the Atmosphere (NUMA), SIAM J. Sci. Comp., 35, B1162-B1194, https://doi.org/10.1137/120876034, 2013.

Guerra, J. E. and Ullrich, P. A.: A high-order staggered finite-element vertical discretization for non-hydrostatic atmospheric models, Geosci. Model Dev., 9, 2007-2029, https://doi.org/10.5194/gmd-9-2007-2016, 2016.

Higueras, I.: Strong stability for Additive Runge-Kutta methods, SIAM J. Numer. Anal., 44, 1735-1758, https://doi.org/10.1137/040612968, 2006.

Higueras, I.: Characterizing Strong Stability Preserving Additive Runge-Kutta Methods, J. Sci. Comput., 39, 115-128, https://doi.org/10.1007/s10915-008-9252-2, 2009.

Higueras, I., Happenhofer, N., Koch, O., and Kupka, F.: Optimized strong stability preserving IMEX Runge-Kutta methods, J. Comput. Appl. Math., 272, 116-140, 2014.

Hindmarsh, A. C., Brown, P. N., Grant, K. E., Lee, S. L., Serban, R., Shumaker, D. E., and Woodward, C. S.: SUNDIALS: Suite of nonlinear and differential/algebraic equation solvers, ACM Trans. Math. Software, 31, 363-396, 2005.

Kennedy, C. A. and Carpenter, M. H.: Additive Runge-Kutta schemes for convection-diffusion-reaction equations, Appl. Numer. Math., 44, 139-181, https://doi.org/10.1016/S01689274(02)00138-1, 2003.

Kinnmark, I. P. and Gray, W. G.: One Step Integration Methods of Third-Fourth Order Accuracy with Large Hyperbolic Stability Limits, Mathematics and Computers in Simulation XXVI, 181$188,1984$.

Klemp, J. B., Skamarock, W. C., and Dudhia, J.: Conservative Split-Explicit Time Integration Methods for the Compressible Nonhydrostatic Equations, Mon. Weather Rev., 135, 2897-2913, https://doi.org/10.1175/MWR3440.1, 2007.

Lock, S.-J., Wood, N., and Weller, H.: Numerical analyses of Runge-Kutta implicit-explicit schemes for horizontally explicit, vertically implicit solutions of atmospheric models, Q. J. Roy. Meteor. Soc., 140, 1654-1669, https://doi.org/10.1002/qj.2246, 2014.

Pareschi, L. and Russo, G.: Implicit-explicit Runge-Kutta schemes and applications to hyperbolic systems with relaxation, J. Sci. Comput., 25, 129-155, https://doi.org/10.1007/BF02728986, 2005.

Reynolds, D. R., Gardner, D. J., Hindmarsh, A. C., Woodward, C. S., and Sexton, J. M.: ARKode - a solver for stiff, nonstiff and mixed systems of ODEs, in preparation, 2018.

Saad, Y. and Schultz, M. H.: GMRES: A generalized minimal residual algorithm for solving nonsymmetric linear systems, SIAM J. Sci. Stat. Comput., 7, 856-869, 1986.

SUNDIALS: SUite of Nonlinear DIfferential/ALgebraic equation Solvers, available at: http://computation.llnl.gov/projects/ sundials, 2017.

Taylor, M. A. and Fournier, A.: A compatible and conservative spectral element method on unstructured grids, J. Comp. Phys., 229, 5879-5895, 2010.

Ullrich, P. A.: A global finite-element shallow-water model supporting continuous and discontinuous elements, Geosci. Model Dev., 7, 3017-3035, https://doi.org/10.5194/gmd-7-3017-2014, 2014.

Ullrich, P. A. and Jablonowski, C.: Operator-Split RungeKutta-Rosenbrock Methods for Nonhydrostatic Atmospheric Models, Mon. Weather Rev., 140, 1257-1284, https://doi.org/10.1175/MWR-D-10-05073.1, 2012.

Ullrich, P. A., Jablonowski, C., Kent, J., Lauritzen, P. H., Nair, R. D., and Taylor, M. A.: Dynamical Core Model Intercomparison Project (DCMIP) 2012 Test Case Document, v.1.7, available at: https://earthsystemcog.org/site_media/docs/ DCMIP-TestCaseDocument_v1.7.pdf, 2012.

Ullrich, P. A., Melvin, T., Jablonowski, C., and Staniforth, A.: A proposed baroclinic wave test case for deep-and shallowatmosphere dynamical cores, Q. J. Roy. Meteor. Soc., 140, 1590$1602,2014$.

Weller, H., Lock, S.-J., and Wood, N.: Runge-Kutta IMEX schemes for the Horizontally Explicit/Vertically Implicit (HEVI) solution of wave equations, J. Comp. Phys., 252, 365-381, https://doi.org/10.1016/j.jcp.2013.06.025, 2013.

Yang, C. and Cai, X.-C.: A Scalable Fully Implicit Compressible Euler Solver for Mesoscale Nonhydrostatic Simulation of Atmospheric Flows, SIAM J. Sci. Comp., 36, S23-S47, https://doi.org/10.1137/130919167, 2014. 
Yang, C., Xue, W., Fu, H., You, H., Wang, X., Ao, Y., Liu, F., Gan, L., Xu, P., Wang, L., Yang, G., and Zheng, W.: 10M-core scalable fully-implicit solver for nonhydrostatic atmospheric dynamics, SC'16: Proceedings of the International Conference for High Performance Computing, Networking, Storage and Analysis, Salt Lake City, UT, USA, 13-18 November 2016, https://doi.org/10.1109/SC.2016.5, 2016. 